Portland State University

PDXScholar

Civil and Environmental Engineering Faculty

Publications and Presentations

$11-1-1991$

\title{
Green's Law Revisited: Tidal Long-Wave Propagation in Channels with Strong Topography
}

David A. Jay

Portland State University

Follow this and additional works at: https://pdxscholar.library.pdx.edu/cengin_fac

Part of the Civil and Environmental Engineering Commons

Let us know how access to this document benefits you.

\section{Citation Details}

Jay, D. A. (1991), Green's law revisited: Tidal long-wave propagation in channels with strong topography, J. Geophys. Res., 96(C11), 20585-20598, doi:10.1029/91JC01633.

This Article is brought to you for free and open access. It has been accepted for inclusion in Civil and Environmental Engineering Faculty Publications and Presentations by an authorized administrator of PDXScholar. Please contact us if we can make this document more accessible: pdxscholar@pdx.edu. 


\title{
Green's Law Revisited: Tidal Long-Wave Propagation in Channels With Strong Topography
}

\author{
D. A. JAY \\ Geophysics Program, University of Washington, Seattle
}

\begin{abstract}
Green's Law states that tidal long-wave elevation $\zeta$ and tidal transport $Q$ vary with width $b$ and depth $h$ according to $\zeta \cong b^{-1 / 2} h^{-1 / 4}$ and $Q \cong b^{+1 / 2} h^{+1 / 4}$. This solution is of limited utility because it is restricted to inviscid, infinitesimal waves in channels with no mean flow and weak topography (those with topographic scale $L \gg$ wavelength $\lambda$ ). An analytical perturbation model including finite-amplitude effects, river flow, and tidal flats has been used to show that (1) wave behavior to lowest order is a function of only two nondimensional parameters representing, respectively, the strength of friction at the bed and the rate of topographic convergence/divergence; (2) two different wave equations with nearly constant coefficients can be derived that together cover most physically relevant values of these parameters, even very strong topography; (3) a single, incident wave in a strongly convergent or divergent geometry may mimic a standing wave by having $a \cong 90^{\circ}$ phase difference between $Q$ and $\zeta$ and a very large phase speed, without the presence of a reflected wave; (4) channels with strong friction and/or strong topography $(L<\lambda)$ show very large deviations from Green's Law; and (5) these deviations arise because both frictional damping and the direct dependence of $|Q|$ and $|\zeta|$ on topography (topographic funnelling) must be considered.
\end{abstract}

\section{INTRODUCTION}

Long-wave propagation in narrow channels of variable width and depth has been the subject of analysis for more than 150 years. One of the first major results was that of Green [1837], after whom Green's functions were named. Green's Law states that the wave amplitude $\zeta$ in a frictionless channel with slowly changing section varies as $b^{-1 / 2} h^{-1 / 4}$, where $b$ is the width (more exactly the width of the momentum-conveying flow or stream width) and $h$ is the depth below a mean tidal datum. Tidal transport $Q$ varies as $b^{+1 / 2} h^{+1 / 4}$. All tidal channel flows are strongly frictional, most are short relative to the tidal wavelength $\lambda$, and their topographic scale $L$ is often much less than the length of the channel. Green's Law is therefore not directly applicable to tidal flows. Most subsequent treatments of wave propagation have at least implicitly followed Green and assumed weak topographic variation $(L \gg \lambda)$. Only Lighthill [1978] and Prandle and Rahman [1980] have explicitly considered the influence of the topographic convergence rate on wave propagation. The former, however, treated inviscid waves exclusively, and inviscid waves do not exist in the strong topography limit. Dronkers [1964], Parker [1984], and Godin [1991] have all focused on the role of frictional nonlinearities in tidal propagation and overtide generation. LeBlond [1978] and C. Friedrichs and O. Madsen (manuscript in preparation, 1991) have examined the "diffusive" behavior of strongly frictional waves in a channel of uniform depth and width. The latter included tidal flats in the analysis. None of these studies explored the very different character of frictional wave propagation over strong topography or the interaction of topography and friction.

The present analysis then focuses on understanding the principles of wave propagation in typical river estuaries and narrow embayments. Friction is important in these systems, and topography may vary along the length of a single estuary on scales from much less than to much greater than a tidal wavelength. The one-dimensional wave equation governing finite-amplitude

1991 by the American Geophysical Union.

91JC01633.

$1 / 91 \mathrm{JC}-01633 \$ 05.00$ long-wave propagation in channels is nonlinear and has not been solved exactly, but two approximate solutions are derived herein. Both include river flow, finite amplitude, friction, and topography. Together, these solutions are adequate to represent wave propagation through narrow channels with arbitrarily strong (but smooth) depth and width variation. One, the "standard solution," is a generalization of Green's solution. It has asymptotically constant coefficients in two circumstances. Either friction and acceleration effects on the wave number are both weak relative to topography (strong topography), or friction and topography are weak relative to acceleration effects (weak topography). The other, the "critical solution," represents wave propagation when the flow is dominated by bed friction. This solution becomes exact (constant coefficient) at a particular, critical convergence rate where acceleration and topographic effects are equal and of opposite sign in the wave equation. Critical convergence is also the greatest possible convergence rate for inviscid waves. Above critical convergence (in the supercritical range), inviscid disturbances damp exponentially rather than propagate [Lighthill, 1978]. Thus friction must be included in any representation of long-wave propogation over strong topography.

Both of these solutions are derived from a perturbation expansion analysis of the long-wave equation. The strength of finiteamplitude nonlinearities (those that give rise to overtide and residual circulations and increase wave propagation speed) in this equation is scaled by the barotropic nonlinearity parameter $\varepsilon=\zeta / H$, the ratio of wave amplitude to mean depth [Stokes, 1847]. In the presence of bed friction, finite-amplitude effects appear not only in the convective acceleration term in the wave equation, but also in the friction term itself [Parker, 1984]. Although it may approach unity in very shallow systems, $\varepsilon$ is small but finite in most systems and is thus appropriate as a perturbation expansion parameter for finite-amplitude effects. The other likely form of analysis for this problem, that based on characteristics, is not particularly appropriate, because at critical convergence the wave equation is diffusive, not hyperbolic, in the terminology of Whitham [1974] and LeBlond [1978].

Finite amplitude is not the only source of nonlinearity in long-wave propagation. Polynomial representation of the bed stress term $c_{D}|U| U$ (where $c_{D} \cong O\left(3 \times 10^{-3}\right)$ is the drag 
coefficient and $U$ is along-channel velocity) gives rise to terms as high as cubic in $U$ [Dronkers, 1964] or even quintic [Godin and Gutierrez, 1986]. River flow acts through the convective accelerations to slow movement of the incident, landward propagating wave and speed that of the reflected, seaward propagating wave in a convergent channel. It also greatly augments the linear and nonlinear effects of friction. Finally, tidal flats adjacent to a channel cause nonlinear effects through the convective acceleration term in the wave equation [Speer and Aubrey, 1985; Friedrichs and Aubrey, 1988]. Momentum is lost on the rising tide as water pours out over flats and decelerates because of strong friction. A further loss occurs on the falling tide as water with essentially zero momentum returns to the channel and must be accelerated. It is thus essential to distinguish between the momentum-conveying width $b$ and the total width $B_{T}$, which also includes the tidal flats.

It will be shown that the primary effects of all these nonlinearities are proportional to $\varepsilon$. Representation of all major nonlinearities in long-wave propagation by a perturbation expansion in a single parameter allows this problem to be carried to $O(\varepsilon)$ in a consistent and straightforward way. Conversely, without carrying representation of the tidal wave itself (and not just the overtide and residual flows) to $O(\varepsilon)$, it is impossible to represent vital features such as finite amplitude and river flow effects on phase speed. The computational version of this perturbation model is one-dimensional, cross-sectionally integrated, and harmonic. It calculates, in addition to river flow and the basic tidal flow, the Stokes drift and tidal residual transport and elevation related thereto, though these are not discussed here.

The topography of most estuaries is sufficiently complex that any realistic representation thereof requires division of the estuary into segments. Each segment may, after the fashion of a finite difference model, be taken constant in width and depth following Dronkers [1964] and many subsequent investigators. Unfortunately, this procedure eliminates the very large effect of convergence/divergence in $b$ and $h$ on the complex wave number $q$ and thus on wave speed $c$. Therefore $b$ and $h$ in an analytical model must be represented by some simple mathematical function; linear, geometric, and exponential functions have been used in the past. Linear or geometric functions allow development of a wave equation that can be transformed to a Bessel's equation [Perroud, 1958; Dorrestein, 1961; and Prandle and Rahman, 1980]. This method has not been used in part because it does not allow inclusion of all relevant features. A transformation of the wave equation using an exponential representation of $b, b_{T}$, and $h$ (where $b_{T}$ is the total mean width, or stream width plus mean tidal flat width) within each segment is used instead. This approach includes all important nonlinearities, and the resulting wave equation is more accessible to qualitative interpretation than with a Bessel's equation approach. The effects of variations in both $b$ and $h$ on wave propagation may be expressed in a single parameter, just as with a Bessel's equation transformation [Prandle and Rahman, 1980].

The analysis that follows includes derivation of a wave equation, determination of a dispersion relationship, and an analysis of the effects of friction and topography on tidal long-wave propagation, culminating in a more general form for Green's law. Results show that the commonly observed $\cong 45^{\circ}$ phase difference between surface elevation $\zeta$ and transport $Q$ in rivers and estuaries may be due to near-critically convergent channel width. That is, the rate of channel convergence is such that the acceleration contribution to complex wave number $q$ is canceled by the convergence effect, so that the sum of these two terms is small relative to the friction term. In convergent/divergent channels, the phase difference incident wave alone may approach $90^{\circ}$, and wave cele impedance $I$ (the ratio of $\zeta$ to $Q$ ) become large. strong topography, an incident wave alone (i.e., reflected wave) mimics a standing wave with incic reflected waves of equal amplitude. The model also Green's Law is greatly modified by the presence and/or strong topography. Finally, finite amplitude wave celerity by a factor of $1+(3 \varepsilon / 2)\left(b_{T} / b\right)$. This the classical result of $1+(3 \varepsilon / 2)$ [Lamb, 1932] for $b_{T}$

Application of this model to actual estuarine topog not discussed because multiple tidal constituents and are vital to tidal predictions and because the intricacies topographies would obscure rather than illuminate features of wave behavior. This analysis concentrates a very general analysis of the response of the fundame alone to topography and friction. The overtide wa included in an extended version of the model) exhi complex behavior because of its nonlinear forcing. No a careful comparison has been made of the present $m$ dictions for the geometry of the Columbia River estua tidal height observations and results from a multic model [Giese and Jay, 1989] for three river flow neap, spring, and mean tides. The performance of th model for $M_{2}$ is systematically better than that of Giese primarily because finite-amplitude and topographic effe wave number have been included here.

\section{GOVERNING EQUATIONS}

\section{Derivation of a Wave Equation}

The first step in any analysis of wave propagation in nels is to derive a one-dimensional wave equation fron ally integrated expressions for conservation of mass a channel momentum. Use of sectionally integrated eq justified because channel curvature, the ratio of widt wavelength, and the Kelvin number (width divided by Rossby radius) are all small in the channels considered The tidal flow is nearly two-dimensional in the along-ch and vertical $(z)$ directions, allowing cross-channel inte the continuity and momentum equations. Tidal transport is a "natural" variable in a stratified tidal flow in a way velocity $U(x, z, t)$ is not, because of the large flood/ebb in vertical distribution of the horizontal tidal flow that many systems [Jay, 1991]. Thus a vertically integrated in which only $Q$ and elevation $\zeta(x, t)$ are calculated is ate. The cross-sectionally integrated dimensional equ conservation of mass and along-channel momentum are

$$
\begin{gathered}
\frac{\partial \hat{Q}}{\partial \hat{x}}+\hat{b} \frac{\partial \mathcal{\zeta}}{\partial \hat{t}}=0 \\
\frac{\partial \hat{Q}}{\partial \hat{t}}+\frac{\partial}{\partial \hat{x}}\left[\frac{\hat{Q}^{2}}{\hat{A}}\right]+g \hat{A} \frac{\partial \mathcal{\zeta}}{\partial \hat{x}}+\hat{b} \hat{T}_{b}=0
\end{gathered}
$$

where

$\hat{x} \quad$ along-channel distance, positive landward;

$\hat{t}$ time;

$\hat{A} \quad$ channel cross-section, equal to $\hat{b}(\hat{h}+\hat{\zeta})$;

$g$ acceleration due to gravity;

$\hat{T}_{b} \quad$ bed stress divided by water density.

A circumflex indicates a dimensional variable. 
Inclusion of tidal flats in a one-dimensional analysis requires that (1) and (2) be modified to account for the momentum flux to and from the tidal flats. This can be accomplished using the ideas of Speer [1984]. Speer assumed that no momentum is conveyed in the $\hat{x}$ direction by flow over these flats. Water moving over the flats on a rising tide loses its momentum to bed friction, so that upon return to the channel as the water level falls, its velocity is zero; it must then be accelerated to the ambient flow velocity. Moreover, the dissipation over the flats is assumed to be small relative to that which occurs in the channels. Clearly, these two assumptions become inconsistent if the area of tidal flats is too large. However, because velocities are typically smaller along the channel margins that feed water to the tidal flats than in midchannel, the following analysis will allow the tidal flats at each $\hat{x}$ to be as wide as the channel itself. C. Friedrichs and O. Madsen (manuscript in preparation, 1991) have considered the effects of arbitrarily wide tidal flats in a channel of constant width and depth with strong friction. This analysis, which they refer to as "kinematic" or "zero inertia," corresponds roughly to the critical convergence case in a channel with uniform (in $\hat{x}$ ) width and depth, but with wide tidal flats; $\varepsilon$ is modified by the presence of these flats.

Consider first the effects of tidal flats on conservation of mass (equation (1)). Define the time-varying total width $\hat{B}_{T}(\hat{x}, \hat{t})$ as the sum of a constant (in time) stream width $\hat{b}(\hat{x})$ and a tidal flat width $\Delta \hat{B}(\hat{x}, \hat{t})$ that varies with the elevation of the tide (Figure 1). Intuitively, it is obvious that the along-channel divergence of $\hat{Q}$ is related to $\hat{B}_{T}$ and not $\hat{b}$, because the surface elevation $\hat{\zeta}$ rises over the entire width, not just the stream width. More formally [Speer, 1984], the presence of tidal flats causes an additional momentum flux $\hat{q}_{e}=\Delta \hat{B} \partial \mathcal{\zeta} / \partial \hat{t}$. Adding the additional momentum flux and assuming a linear tidal flat profile, conservation of mass (1) may be written

$$
\begin{aligned}
0=\frac{\partial \hat{Q}}{\partial \hat{x}}+\hat{B}_{T} \frac{\partial \zeta}{\partial \hat{t}} & =\frac{\partial \hat{Q}}{\partial \hat{x}}+\hat{b}_{T} \frac{\partial \hat{\zeta}}{\partial \hat{t}}+\frac{\Delta \hat{b}}{2|\hat{\zeta}|} \frac{\partial\left(\xi^{2}\right)}{\partial \hat{t}} \\
& \cong \frac{\partial \hat{Q}}{\partial \hat{x}}+\hat{b}_{T} \frac{\partial \hat{\zeta}}{\partial \hat{t}}
\end{aligned}
$$

at tidal frequency, where $|\hat{\zeta}|$ is the tidal amplitude. Tidal flats have thus two effects on mass conservation. First, $\hat{b}_{T}$ rather than $\hat{b}$ appears as the multiplier of $\partial \hat{\zeta} / \partial \hat{t}$, and second, a new nonlinear term has appeared that is associated with the tidal cycle variation in tidal flat width. The latter term is important to overtide generation but will not influence the basic tidal motion. Friedrichs et al. [1990] have argued that, for a wide tidal flat, the form of the tidal flat profile is important because this profile changes the amount of storage over the flats. Any of several different tidal flat profiles could be incorporated in the present analysis. Use of the simplest possible (linear) profile herein minimizes algebraic complexity and is consonant with the generally poor knowledge of tidal flat profiles in most estuaries.

The effect of the momentum flux associated with flow of water on and off tidal flats in the along-channel momentum equation is the product of change in velocity $-\hat{Q} / \hat{A}$ as water moves onto the flats times the tidal flat momentum flux $\hat{q}_{e}$ [Speer, 1984]. With some further manipulation and taking $\Delta \hat{B} / \hat{B}_{T} \cong \Delta \hat{b} / \hat{b}_{T}$, (2) may be written

$$
\begin{gathered}
\frac{\partial \hat{Q}}{\partial \hat{t}}+\frac{2 \hat{b}_{T}-\hat{b}}{\hat{b}_{T}} \frac{\partial}{\partial \hat{x}}\left[\frac{\hat{Q}^{2}}{\hat{A}}\right]-\frac{\hat{b}_{T}-\hat{b}}{\hat{b}_{T}} \hat{Q} \frac{\partial}{\partial \hat{x}}\left[\frac{\hat{Q}}{\hat{A}}\right] \\
+g \hat{A} \frac{\partial \hat{\zeta}}{\partial \hat{x}}+\hat{b} \hat{T}_{b}=0
\end{gathered}
$$

Thus as one intuitively expects, the momentum flux associated with the presence of tidal flats modifies the convective acceleration terms in the equation of motion.

The above two first-order differential equations in $\hat{Q}$ and $\zeta$ representing conservation of mass and momentum, (3) and (4), may be combined by cross differentiation to form a second-order wave equation in one variable. In principle, it is immaterial whether this wave equation is in $\hat{Q}$ or $\zeta$. However, the approximate form of the bed stress term to be used herein is much simpler if the wave equation is formed in $\hat{Q}$. Thus $\partial / \partial \hat{x}$ of $(3)$ is subtracted from $\partial / \partial \hat{t}$ of (4), and (3) is used to express the pressure gradient in terms of $\hat{Q}$. Several bilinear pressure gradient terms are neglected because they do not effect the tidal motion before $O\left(\varepsilon^{2}\right)$. At the same time, two simplifications are made in the convective accelerations. First, the only tidal-frequency terms in the bilinear convective accelerations are those involving $\hat{Q}_{R} \hat{Q} / \hat{A}=$ $\hat{U}_{R} \hat{Q}$, where $\hat{Q}_{R}$ is the river flow transport and $\hat{U}_{R}$ is the river

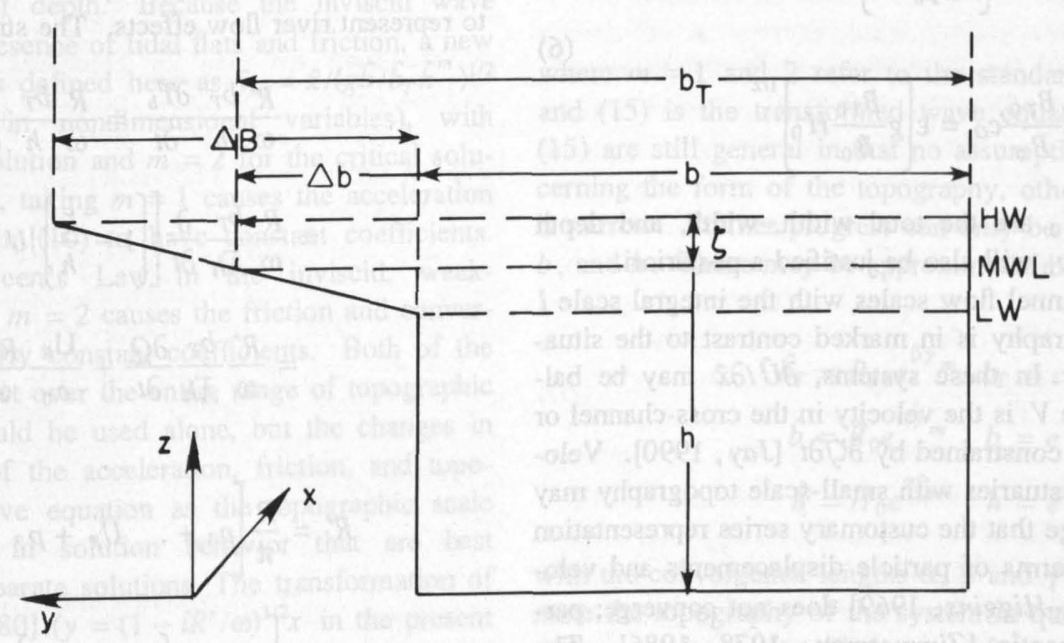

Fig. 1. Model geometry, with MWL as mean water level, HW as high water, $\mathrm{LW}$ as low water, $\Delta B(x, t)$ as time-variable tidal flat width, $\Delta b(x)$ as mean tidal flat width, $b$ as momentum conveying width, $b_{T}$ as total width, $h$ as mean depth, and $\zeta$ as tidal elevation. The positive $x$ direction is landward. 
flow velocity. River flow is assumed steady and enters the system only at the upstream end, so that $\hat{Q}_{R}$ is invariant with $\hat{x}$ and $\hat{t}$. Second, because the convective accelerations will ultimately scale as $O(\varepsilon), \bar{A}=\hat{h} \hat{b}$ rather than $\hat{A}=\hat{b} \hat{h}(1+\zeta / \hat{h})$ may be used in the convective accelerations. The neglected terms in $\xi / \hat{h}$ are $O(\varepsilon)$ relative to the remaining terms and are thus only $O\left(\varepsilon^{2}\right)$.

The resulting dimensional wave equation is

$$
\begin{gathered}
\frac{\partial^{2} \hat{Q}}{\partial \hat{x}^{2}}-\frac{1}{\hat{b}_{T}} \frac{d \hat{b}_{T}}{d \hat{x}} \frac{\partial \hat{Q}}{\partial \hat{x}}-\frac{3 \hat{b}_{T}-\hat{b}}{g \hat{A}} \hat{U}_{R} \frac{\partial^{2} \hat{Q}}{\partial \hat{x} \partial \hat{t}} \\
+\frac{2 \hat{b}_{T}}{g \hat{A}^{2}} \hat{U}_{R} \frac{d \hat{A}}{d \hat{x}} \frac{\partial \hat{Q}}{\partial \hat{t}}-\frac{\hat{b}_{T}}{g \hat{A}} \frac{\partial^{2} \hat{Q}}{\partial \hat{t}^{2}}-\frac{\hat{b}_{T}}{g \hat{h}} \frac{\partial \hat{T}_{b}}{\partial \hat{t}}=0
\end{gathered}
$$

The first term on the left-hand side of (5) arises from the pressure gradient, the second is from the pressure gradient and topography, the following two are convective accelerations, the next is the acceleration term and the last is the bed stress.

\section{Scaling the Wave Equation}

The most important issue in scaling the wave equation (5) is to determine the magnitude of $\partial \hat{Q} / \partial \hat{x}=\hat{A} \partial \hat{U} / \partial \hat{x}$. For weak topography and no tidal flats this is customarily done from (3) under the assumption that the scale for $\hat{x}$ is the inverse wave number $l=\lambda(2 \pi)$. The result is that the along-channel velocity scale $U$ is $\varepsilon c \cong \varepsilon(g \hat{h})^{1 / 2}$. Intuitively, one would expect that topography of a scale $L<l$ would cause $\partial \hat{U} / \partial \hat{x}$ to increase. However, the magnitude of $\partial \hat{U} / \partial \hat{x}$ in a channel flow is constrained by that of $\partial \hat{\zeta} / \partial \hat{t}$ which is fixed by the tidal frequency (equation (3)). Thus while topography creates turbulence and may enhance overtide generation, the velocity $\hat{U}_{L}$ associated with topography of scale $L$ at any given tidal frequency must vary with as $L / l \mathrm{U}$ so that $\partial \hat{U} / \partial \hat{x}$ is independent of $L / l$. The scaling used in the weak topography limit $(L \gg l)$ is thus appropriate for all $L$; i.e., $\partial \hat{U} / \partial \hat{x}=U / l$. The velocity for the $n^{\text {th }}$ overtide will then scale as $\varepsilon^{n} U$ in the usual manner. A simple modification of the above scaling is needed to take account for the presence of tidal flats. From (3) and the spreading of wave crests over the tidal flats:

$$
\begin{gathered}
c_{0}=\left[g \frac{B_{0}}{B_{T 0}} H_{0}\right]^{1 / 2} \\
U=\varepsilon \frac{B_{T 0}}{B_{0}} c_{O}=\varepsilon\left[g \frac{B_{T 0}}{B_{0}} H_{0}\right]^{1 / 2}
\end{gathered}
$$

where $B_{T 0}, B_{0}$, and $H_{0}$ are the total width, width, and depth scales. This choice of $c_{0}$ will also be justified a posteriori.

That $\partial \hat{U} / \partial \hat{x}$ in a channel flow scales with the integral scale $l$ regardless of the topography is in marked contrast to the situation in wide estuaries. In these systems, $\partial \hat{U} / \partial \hat{x}$ may be balanced by $\partial \hat{V} / \partial \hat{y}$ (where $V$ is the velocity in the cross-channel or $y$ direction) and is not constrained by $\partial \zeta / \partial t$ [Jay, 1990]. Velocity gradients in wide estuaries with small-scale topography may become sufficiently large that the customary series representation of the Stokes drift in terms of particle displacements and velocity gradients [Longuet-Higgins, 1969] does not converge; particle drifts become chaotic [Zimmerman, 1978, 1986]. The present analysis cannot be applied to such systems.

The scaling used to nondimensionalize (4) is

$$
\begin{array}{cc}
\hat{Q}=\mathrm{U} B_{0} H_{0} Q & \hat{U}_{R}=\mathrm{U}_{R} U_{R} \\
\hat{b}=B_{0} b & \hat{h}=H_{0} h \quad \hat{b}_{T}=B_{T 0} b_{T} \\
\hat{T}_{b}=c_{D} \mathrm{U}^{2} T_{b} & \mathrm{U}=\varepsilon c_{0} \\
\hat{x}=b x & \hat{t}=\frac{t}{\omega}
\end{array}
$$

The resulting nondimensional wave equation is

$$
\begin{gathered}
\frac{\partial^{2} Q}{\partial x^{2}}-\frac{R}{\omega} \frac{b_{T}}{h} \frac{\partial T_{b}}{\partial t}-\frac{1}{b_{T}} \frac{d b_{T}}{d x} \frac{\partial Q}{\partial x} \\
-\frac{\mathrm{U}_{R}}{c_{0}}\left[\frac{3 b_{T}-b}{b}\right] \frac{U_{R}}{h} \frac{\partial^{2} Q}{\partial x \partial t}-\frac{b_{T}}{\bar{A}} \frac{\partial^{2} Q}{\partial t^{2}} \\
+2 \frac{\mathrm{U}_{R}}{c_{0}} \frac{b_{T}}{\bar{A}} \frac{U_{R}}{\bar{A}} \frac{d A}{d x} \frac{\partial Q}{\partial t}=0
\end{gathered}
$$

where $R=c_{D} /(\mathrm{U} H)$. The nondimensional number $\mathrm{U}_{R} / c_{0}$ multiplying the two convective acceleration terms can also be written $\varepsilon U_{R} / U$, indicating that the convective acceleration is small relative to the remaining terms. The ratio $R / \omega$ is a measure of the relative strength of friction and acceleration in the wave equation. This varies with the frequency of tidal wave in question but is typically $O(1)$ for the major tidal constituents. Thus the present scaling differs from that of LeBlond [1978], where the acceleration terms were taken to be small. Nonetheless, it will be shown that friction often dominates wave propagation in river estuaries because the effects of acceleration on the wave number are balanced by those of channel convergence. A solution to this form of the wave equation should give $Q$ for the basic tidal motion correct to $O(\varepsilon)$.

The next step in the analysis is representation of the bed stress. The harmonic model formulated herein requires that the quadratic stress term $T_{b}=c_{D}|U| U$ be replaced by some approximate form that avoids the need to determine the time of the zero crossings of $U$. A number of schemes have been used for this purpose, as reviewed by Godin [1991]. Most common is a Fourier development introduced by Proudman [1953]. This approach suffers from the shortcomings that it does not introduce even overtides ( $M_{6}$ and the like) and cannot directly represent the river flow. The Tschebyschev polynomial approach of Dronkers [1964] is used here because of the need to represent river flow effects. The stress is given by

$$
\begin{aligned}
& \frac{R}{\omega} \frac{b_{T}}{h} \frac{\partial T_{b}}{\partial t}=\frac{R}{\omega} \frac{b_{T}}{h} \frac{\partial}{\partial t}[|U| U] \\
= & \frac{R}{\omega} \frac{b_{T}}{\bar{A} h} \frac{\partial}{\partial t}\left[\left[1-\frac{\zeta}{h}\right]|U| U \bar{A}\right]+0\left(\varepsilon^{2}\right) \\
= & \frac{R^{\prime}}{\omega} \frac{b_{T}}{\bar{A} h} \frac{\partial Q}{\partial t}+\frac{\mathrm{U}_{R}}{c_{0}} \frac{R^{\prime \prime}}{\omega} \frac{Q_{R}}{\bar{A} h^{2}} \frac{\partial Q}{\partial x} \\
R^{\prime}= & \frac{R}{\pi}\left[p_{1}+. \quad U_{R}+p_{3}\left[3 U_{R}^{2}+\frac{1}{2} U_{a}^{2}\right)\right] \\
R^{\prime \prime}= & \frac{R}{\pi}\left[p_{1}+p_{2}\left[U_{R}+\frac{U_{a}^{2}}{U_{R}}\right]+p_{3}\left[U_{R}^{2}+\frac{3}{2} U_{a}^{2}\right)\right]
\end{aligned}
$$


where $U_{a}$ is the amplitude of $U$, and the time-varying area introduces a finite amplitude nonlinearity. The $p_{j}$ are Tschebyschev coefficients. They are functions of the ratio $U_{R} / U$ and therefore vary along the channel. For $U_{R} / U=0, p_{2}=0$ and $p_{1}=8 / 3$, as in Proudman's [1953] linearization. Far upriver where the current never reverses because the river flow is greater than the tidal flow and $U_{R} / U \geq 1, p_{1}=p_{3}=0$ and $p_{2}=\pi$ so that $|U| U=U_{R} U$. The $p_{3}$ term is responsible for the $O(1)$ interaction of tidal constituents. Each additional transport $Q_{k}$ for constituent $k$ adds to $R^{\prime} / \omega$ a term of the form $U_{a k}^{2} p_{3} / 2$, where $U_{a k}$ is the velocity amplitude for constituent $k$. The present treatment is similar to that of Godin and Gutierrez [1986] for $Q_{R} / Q=0$. They suggest for analytical purposes $|U| U=0.5\left(m U+1 / m U^{3}\right)$ with $m=0.7$ as an approximation to an error-minimizing form that also contains a term in $U^{5}$.

\section{Approximate Solutions}

Approximate solutions to the wave equation (8) with the stress term given by (9) can be found by assuming a harmonic form for the tidal transport: $Q=\operatorname{Re}\left[P(x) e^{i t}\right]$. The resulting ordinary differential equation is, dropping the overbar on $\bar{A}$,

$$
\begin{gathered}
P_{, \mathrm{xx}}+T(x) P_{, x}+G(x) P=0 \\
G(x)=\left[\frac{b_{T}}{b h}\left[1-\frac{i R^{\prime}}{\omega} \frac{1}{h}+2 i \frac{\mathrm{U}_{R}}{c} \frac{U_{R}}{A} \frac{d A}{d x}\right]\right] \\
T(x)=-\left[\frac{1}{b_{T}} \frac{d b_{T}}{d x}+\frac{i \mathrm{U}_{R}}{c}\left[\frac{3 b_{T}}{b}-1+\frac{R^{\prime \prime}}{\omega} \frac{1}{b h^{2}}\right] \frac{U_{R}}{h}\right]
\end{gathered}
$$

where a subscript following a comma indicates differentiation. Two solutions to (10) will be developed here to span the range of physical situations from strong to weak topography. The first covers both weak and strong convergence $(m=1$, the standard solution), while the second is appropriate to the intermediate, frictionally dominated regime ( $m=2$, the critical solution). Both solutions use a method first developed by Green [1837]: changes first of the independent and then the dependent variable. Green distorted along-channel distance $\hat{x}$ by the inviscid wave speed $(g \hat{h})^{1 / 2}$, so that an inviscid, infinitesimal amplitude wave would move equal distances in the distorted coordinate system in equal time, regardless of depth. Because the inviscid wave speed is altered by the presence of tidal flats and friction, a new independent variable $\hat{y}$ is defined here as $\hat{y}_{m}=\hat{x} /\left(g \hat{b} / \hat{b}_{T} \hat{h}^{m}\right)^{1 / 2}$ (or $y_{m}=x /\left(b / b_{T} h^{m}\right)^{1 / 2}$ in nondimensional variables), with $m=1$ for the standard solution and $m=2$ for the critical solution. As is shown below, taking $m=1$ causes the acceleration and convergence terms in (10) to have constant coefficients. This solution gives Green's Law in the inviscid, weaktopography limit. Taking $m=2$ causes the friction and convergence terms to have nearly constant coefficients. Both of the solutions defined here exist over the entire range of topographic variability, and either could be used alone, but the changes in the relative importance of the acceleration, friction, and topographic terms in the wave equation as the topographic scale varies cause alterations in solution behavior that are best represented using two separate solutions. The transformation of Prandle and Rahman [1980] $\left(y=\left(1-i R^{\prime} / \omega\right)^{1 / 2} x\right.$ in the present notation) incorporates both acceleration and friction effects on wave speed. It is not used here for several reasons. First a factor of $1 / h$ represented explicitly in (9) was included by Prandle and Rahman in $R$. Because of the explicit presence of this factor of $1 / h$, use of their transformation would not in the present analysis lead to a constant coefficient equation. Second, such a transformation defines a solution that does not vary smoothly from weak to strong topography. It has a singular point at the intermediate point of critical convergence (defined below), and yet this critically convergent case is precisely where friction is most important. Third, and more physically, topography, not friction, is the primary factor altering the wave speed from its inviscid value. Recognition of the role of convergence/ divergence in controlling the nature of the solution suggests the use of two transformations defined here.

The change of independent variable causes the wave equation to take the form

$$
\begin{aligned}
& P_{y_{m} y_{m}}+T_{m} P_{y_{m}}+G_{m} P=0 \quad m=1,2 \\
& T_{m}\left(y_{m}\right)=- {\left[\frac{1}{2}\left[\frac{1}{b_{T}} \frac{d b_{T}}{d y_{m}}+\frac{1}{b} \frac{d b}{d y_{m}}+\frac{m}{h} \frac{d h}{d y_{m}}\right]+i \frac{V_{R}}{c} \frac{U_{R}}{h}\right.} \\
&\left.\times\left[\frac{b h^{2-m}}{b_{T}}\right]^{1 / 2}\left[\frac{3 b_{T}}{b}-1+\frac{R^{\prime \prime}}{\omega} \frac{1}{b h^{2}}\right)\right] \\
& G_{m}\left(y_{m}\right)= {\left[h^{m-1}-\frac{i R^{\prime}}{w} \frac{1}{h^{2-m}}+2 i \frac{V_{R}}{c}\left(\frac{b_{T}}{b}\right)^{3 / 2-m} \frac{U_{R}}{A} \frac{d A}{d y_{m}}\right] }
\end{aligned}
$$

The change of dependent variable then yields

$$
\begin{gathered}
P\left(y_{m}\right)=Z\left(y_{m}\right) V\left(y_{m}\right) \\
V_{y_{m} y_{m}}+S_{m}\left(y_{m}\right) V=0 \\
S_{m}\left(y_{m}\right)=G_{m}\left(y_{m}\right)-\frac{1}{4} T_{m}^{2}\left(y_{m}\right)-\frac{1}{2} \frac{d T_{m}\left(y_{m}\right)}{d y_{m}} \\
Z\left(y_{m}\right)=\exp \left(-\frac{1}{2} \int T_{m}\left(y_{m}\right) d y_{m}\right)
\end{gathered}
$$

where $m=1$ and 2 refer to the standard and critical solutions and (15) is the transformed wave equation. Note that (11) to (15) are still general in that no assumption has been made concerning the form of the topography, other than that the channel is narrow. Further progress can only be made by specifying $b_{T}$, $b$, and $h$. These may be approximated by exponentials:

$$
\begin{array}{ll}
\hat{b}_{T}=B_{T 0 e^{\beta g_{m}}} & b_{T}=e^{\beta l y_{m}} \\
\hat{b}=B_{0} e^{\alpha g_{m}} & b=e^{\alpha l y_{m}} \\
\hat{h}=H_{0} e^{\gamma g_{m}} & h=e^{\gamma l y_{m}}
\end{array}
$$

with the convergence lengths $\alpha, \beta$ and $\gamma$ chosen to best approximate the topography of the system in question. Irregular topography may be dealt with by use of a model with multiple segments, each having different topographic characteristics. Substitution of (16) into (14) gives, after some algebra, 


$$
\begin{array}{r}
S_{m}\left(y_{m}\right)=\left[h^{m-1}-\left[\frac{\Delta_{m} l}{2}\right]^{2}-i \frac{R^{\prime}}{w} \frac{1}{h^{2-m}}\right] \\
+i \frac{\mathrm{U}_{R}}{c} \frac{U_{R}}{h}\left[\frac{b}{b_{T} h^{2-m}}\right]^{1 / 2} \\
\times\left[-\frac{b_{T}}{b}\left[\alpha l+\frac{\gamma l}{m^{2}}\right]+\left[\Delta_{m} l+(3-2 m) \gamma l\right]\right. \\
\left.-\frac{R^{\prime \prime}}{w} \frac{1}{b h^{2}}\left\{\alpha l+\frac{\beta l}{2}+\left[2+\frac{m-1}{2}\right] \gamma l\right\}\right]+0\left(\varepsilon^{2}\right)
\end{array}
$$

where $\Delta_{m} l=1 / 2(\alpha+\beta+m \gamma) l$.

The transformed wave equation (15) does not have constant coefficients for either the standard or critical solutions unless $h$ is constant. Even then, along-channel variability of $R^{\prime}$ and thus $S_{m}(y)$ is caused by along-channel changes in river flow and tidal velocity. Still, the $O(1)$ part of (17) is nearly constant coefficient for $m=1$ when the topographic scale $L \gg l$, and, as is discussed below, it also has constant coefficients asymptotically as $L$ goes to zero. For $m=2$, (17) will be shown below to have constant coefficients to $O(1)$ near critical convergence. Thus it is appropriate to derive an approximate solution by dividing our model estuary into segments much less than a wavelength long. Within each of these segments, (15) with (16) and (17) can be used to approximate the true solution. More specifically, because of the strong effect of river flow on $R^{\prime} / \omega$, we require that the segment length $L_{x}$ be less than the $e$-folding length of the topography, i.e., that $L_{x} \ll$ minimum $(1 / \alpha, 1 / \beta, 1 / \gamma)$.

The dimensionless, complex wave number $q_{m} l$ is simply $S_{m}^{1 / 2} l$. The dimensional wave number $q_{m}$ and wave celerity $c_{m}$ in the undistorted coordinate system are given by

$$
\begin{gathered}
q_{m}=\left(k_{m}+i r_{m}\right)=\frac{\omega}{c_{0}} S_{m}^{1 / 2} \\
c_{m}=\left[\frac{b h^{m}}{b_{T}}\right]^{1 / 2} \frac{\omega}{k_{m}}
\end{gathered}
$$

where $k_{m}$, the real part of $q_{m}$, corresponds to the usual inviscid wave number and $r_{m}$, the imaginary part of $q_{m}$, is the damping modulus [Dronkers, 1964]. $V\left(y_{m}\right)$ and $Z\left(y_{m}\right)$ are then

$$
\begin{aligned}
& V\left(y_{m}\right)=A e^{i q_{m} l y_{m}}+B e^{-i q_{m} l y_{m}} \\
& Z\left(y_{m}\right)=\left[b_{T} b h^{m}\right]^{1 / 4} e^{-i \delta_{m} l y_{m}}
\end{aligned}
$$

where $A$ and $B$ are coefficients determined from the boundary conditions and $\delta_{m}$ is a real $O(\varepsilon)$ correction to $k_{m} l$ that is proportional to $U_{R}$ and emerges from integration of the $O(\varepsilon)$ part of $T\left(y_{m}\right)$ in (15). It serves to retard the incident wave and accelerate the reflected wave in proportion to the strength of the river flow.

The complete form of the solutions for $Q$ and $\zeta$ is

$$
\begin{gathered}
Q\left(y_{m}, t\right)=\operatorname{Re}\left[\left(b_{T} b h^{m}\right)^{1 / 4}\right. \\
\left.\times\left[A e^{i\left(q_{m}-\delta_{m}\right) l y_{m}}+B e^{-i\left(q_{m}+\delta_{m}\right) l y_{m}}\right] e^{i t}\right] \\
=R_{e}\left[\left[A e^{c^{+} y_{m}}+B e^{c^{-} y_{m}}\right] e^{i t}\right] \\
\zeta\left(y_{m}, t\right)=R_{e}\left[i\left(b_{T} b h^{m}\right)^{-1 / 4}\right. \\
\left.\left.\times c^{+} e^{i\left(q_{m}-\delta_{m}\right) t y_{m}}+B c^{-} e^{-i\left(q_{m}+\delta_{m}\right) l y_{m}}\right] e^{i t}\right] \\
=\operatorname{Re}\left[i\left[A c^{+} e^{c^{+} y_{m}}+B c^{-} e^{c^{-} y_{m}}\right] e^{i t}\right] \\
c^{+}=\left[\frac{\Delta_{m}}{2}+i\left(q_{m}-\delta_{m}\right)\right] l \\
c^{-}=\left[\frac{\Delta_{m}}{2}-i\left(q_{m}+\delta_{m}\right)\right] l
\end{gathered}
$$

using continuity to determine $\zeta$ from $Q$.

The $\left(b b_{T h}\right)^{1 / 4}=\exp (\Delta l / 2) y_{m}$ and $\left(b b_{T h}\right)^{-1 / 4}=\exp (-\Delta l / 2) y_{m}$ dependences of $Q$ and $\zeta$ for the standard solution $(m=1)$ are for $b=b_{T}$ the same as seen in Green's Law, but this direct topographic dependence or topographic funneling is modified in the presence of friction by the damping modulus $r_{m} l$ which also depends on the topography. As is discussed below, topographic dependence through $r_{m} l$ often dominates over topographic funneling. It is only in the inviscid, weak topography limit that the standard solution reduces to Green's Law. The critical differs from the standard solution not only in the form of the direct topographic dependence of $Q$ and $\zeta$, but also in form of $q_{m} l$.

The final step in solution of the wave equation (11) is application of boundary conditions to determine the constants $A$ and $B$ in (19), which are, respectively, the complex coefficients of the reflected and incident waves for each segment. Each coefficient specifies an amplitude and phase. Division of the system into $n$ segments complicates application of the boundary conditions because there are then $2 n$ constants for the $n$ incident and reflected waves. These $2 n$ constants must be determined from two boundary conditions and $2 n-2$ matching conditions between segments by a Gaussian elimination procedure first used in this context by Dronkers [1964]. The matching conditions are linear and guarantee the continuity of $Q$ and $\zeta$ between segments. This suffices to guarantee conservation of energy as well [Lighthill, 1978]. One boundary condition (amplitude and phase of the wave at the estuary entrance) is applied at $x=y_{m}=0$, while the other is applied at the upstream end. There are two possibilities at the upstream boundary. Either the wave dies out uniformly at large $y_{m}$, or complete reflection occurs at some finite $y_{m}$.

\section{WAVE NUMBER AND WAVE SPEED}

\section{$O$ (1) Properties}

$P\left(y_{m}\right)$, the spatial part of $Q$, depends only on $y_{m}, \Delta_{m} l$ topographic funneling), $q_{m} l$, and $\delta_{m} l ; \delta_{m} l$ is proportional to $U_{R}$ 
and is $O(\varepsilon)$. The $O(1)$ part of $q_{m} l$ depends only on three $O(1)$ terms in $S_{m}$ (17). To lowest order, $q_{m} l$ is given by

$$
q_{m} l=\left[h^{m-1}-\left[\frac{\Delta_{m} l}{2}\right]^{2}-\frac{i R^{\prime}}{\omega} \frac{1}{h^{2-m}}\right]^{1 / 2}+O(\varepsilon)
$$

The leftmost term in $q_{m} l$ comes from the acceleration term in the wave equation (5). For uniform width and depth with $b_{T}=b$ and no friction, (22) gives the usual long-wave results for the dimensional wave number and celerity $q=k=\omega /(g h)^{1 / 2}$ and $c=$ $(g h)^{1 / 2}$ in the undistorted coordinate system. (Henceforth, explicit identification of the standard and critical solutions by subscript will be made only when necessary for clarity. The subscript $m$ on $y, q$, and $S$ will also be dropped.) In the presence of tidal flats, $c=\left(g b / b_{T} h\right)^{1 / 2}$, justifying (6). Inclusion of bed stress in (5) introduces an imaginary part to $q l$ that arises from the right-hand term in (22). The imaginary part of $q l$ is negative for $R^{\prime}>0$. Choosing the root that gives $k l>0$ leads to $r l<0$. Thus both incident and reflected waves damp in their respective directions of propagation. The middle term of $q l$ in (22) is a correction to $q l$ caused by channel convergence/divergence. It always decreases $k l$ and increases $c$.

It is evident from (22) that the $O(1)$ part of $q l$ is a function of $h$ and two nondimensional parameters, $R^{\prime} / \omega$ and $\Delta l$. However, the transformations used in determining the standard and critical solutions are such that $h$ in the $O(1)$ part of each solution appears only in a term which is not dominant, and because model segments are short, $h$ is always $O(1)$. Therefore the $O(1)$ behavior of the solution is actually governed in both models by the two nondimensional parameters. $R^{\prime} / \omega$ and $\Delta l$ are respectively the nondimensional ratios of the importance of friction and topographic convergence/divergence to acceleration in the wave equation. The model of Prandle and Rahman [1980] is controlled by two analogous nondimensional numbers.

A general understanding of the behavior of $q l$ and the incident and reflected waves as a function of the two major nondimensional parameters can be obtained from Figures 2 to 5 . These plots were constructed using the complete form of $S$ from (17), with $\varepsilon=0.1, \mathrm{U}_{R}=\mathrm{U}, U_{R}=0.1, b_{T}=b, \quad \gamma l=0$, and $\Delta_{1} l=\Delta_{2} l=\alpha l<0$ (convergent geometry). Constant depth eliminates the distinction between the two solutions. This does not constitute to $O(1)$ a loss of information, because the dependence of the two models on $\Delta l$ is identical; only the definition of $\Delta l$ changes. Properties for divergent geometries also differ only at $O(\varepsilon)$. Consider first the real and imaginary parts of $q l, k l$ and $r l$ (Figures $2 a$ and $2 b$ ). Using the scaling of (7), $R^{\prime} / \omega$ is $O(1)$ for the major tidal constituents, $<1$ for overtides and $O(10)$ for tidal residual wave components and shelf wave-forced oscillations. Note that $R^{\prime}$ is nearly invariant with frequency, because $R^{\prime}$ for all waves in a system is set primarily by the strongest tidal constituent(s) and river flow, if this is large.

For $|\Delta l|$ very small, $q l \cong\left(1-i R^{\prime} / \omega\right)^{1 / 2} \cong 2^{1 / 4}(\cos \pi / 8+$ $i \sin \pi / 8)$, taking $R^{\prime} / \omega=1$. From Figure $2 a, k l$ is $O(1)$ in this asymptote and only weakly variable over the frequency range of the major tidal species. In the strong-friction (or low frequency) asymptote, however, $k l$ increases (wave speed decreases) rapidly with increasing $R^{\prime} / \omega$. Thus long waves are at least weakly dispersive in the presence of friction. The damping modulus $r l$ is, in contrast, strongly variable at the weak-friction (high frequency) asymptote and continues to increase slowly with $R^{\prime} / \omega$ as the high friction asymptote is approached (Figure $2 b$ ).
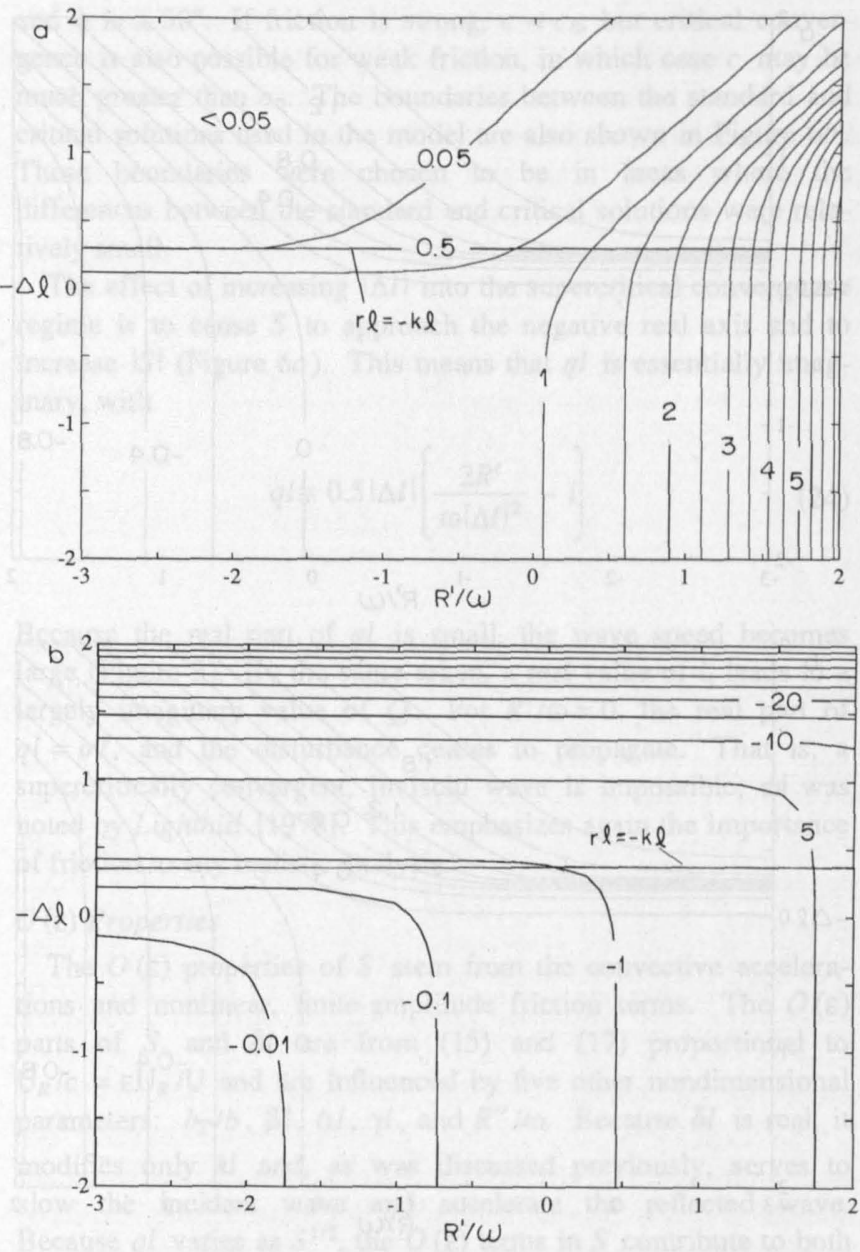

Fig. 2. Components of the complex wave number $q l=(k+i r) l$ for convergent geometry as functions of the logs (to the base 10 here and henceforth) of $R^{\prime} / \omega$ and $-\Delta l$, the two nondimensional numbers that control $O(1)$ wave behavior. $R^{\prime} / \omega$ is the nondimensional bed friction parameter, and $\Delta l$ is the topographic convergence parameter. Figure 2 and other similar plots were constructed for convergent geometry with $\varepsilon=0.1$, river flow $U_{R}=0.1 \mathrm{~m} \mathrm{~s}^{-1}$, and $b_{T}=b$. The depth is constant, so that $\Delta l=\alpha l / 2$. In figure $2 a, k l$ is the wave number in the usual sense, and $r l$ in Figure $2 b$ is the damping modulus, which vanishes in the inviscid limit as $R^{\prime} / \omega$ approaches zero. Critical convergence occurs when $k l=-r l$. Other properties are discussed in the text.

The quadratic dependence of $q l$ on changes in total width and depth (the $\Delta l$ term in (22)) suggest that strong topography, if present, should play a very important role in determining $q l$ and thus $c$. From (22) it can be seen that with $U_{R}=0$ and $m=1$, the acceleration and convergence terms cancel for $|\Delta l|=2$. This $I$ have called critical convergence. (Inclusion of $O(\varepsilon)$ effects causes critical convergence to be a function of $U_{R}$ and to occur for $|\Delta l|$ somewhat less than 2 in Figures $2 a$ and $2 b$.) As $|\Delta l|$ increases beyond critical convergence (supercritical convergence), $\Delta l$ increasingly dominates $k l$ and $r l$. Thus both friction and channel convergence are important in determining wave speed.

The effects of friction and convergence on wave speed for the incident and reflected waves are seen in Figures $3 a$ and $3 b$. (Subscripts $i$ and $r$ indicate here and henceforth the incident and reflected waves.) As long as convergence is subcritical $(|\Delta l|<2)$, friction controls $c / c_{0}$ for both incident and reflected waves. For $R^{\prime} / \omega \ll 1, c / c_{0}$ is slightly greater than 1 because of the effect of finite amplitude in accelerating the wave, as is discussed below. For $R^{\prime} / \omega O(1)$ the frictional retardation of the tidal wave is less than $10 \%$. This is the same order and opposite 

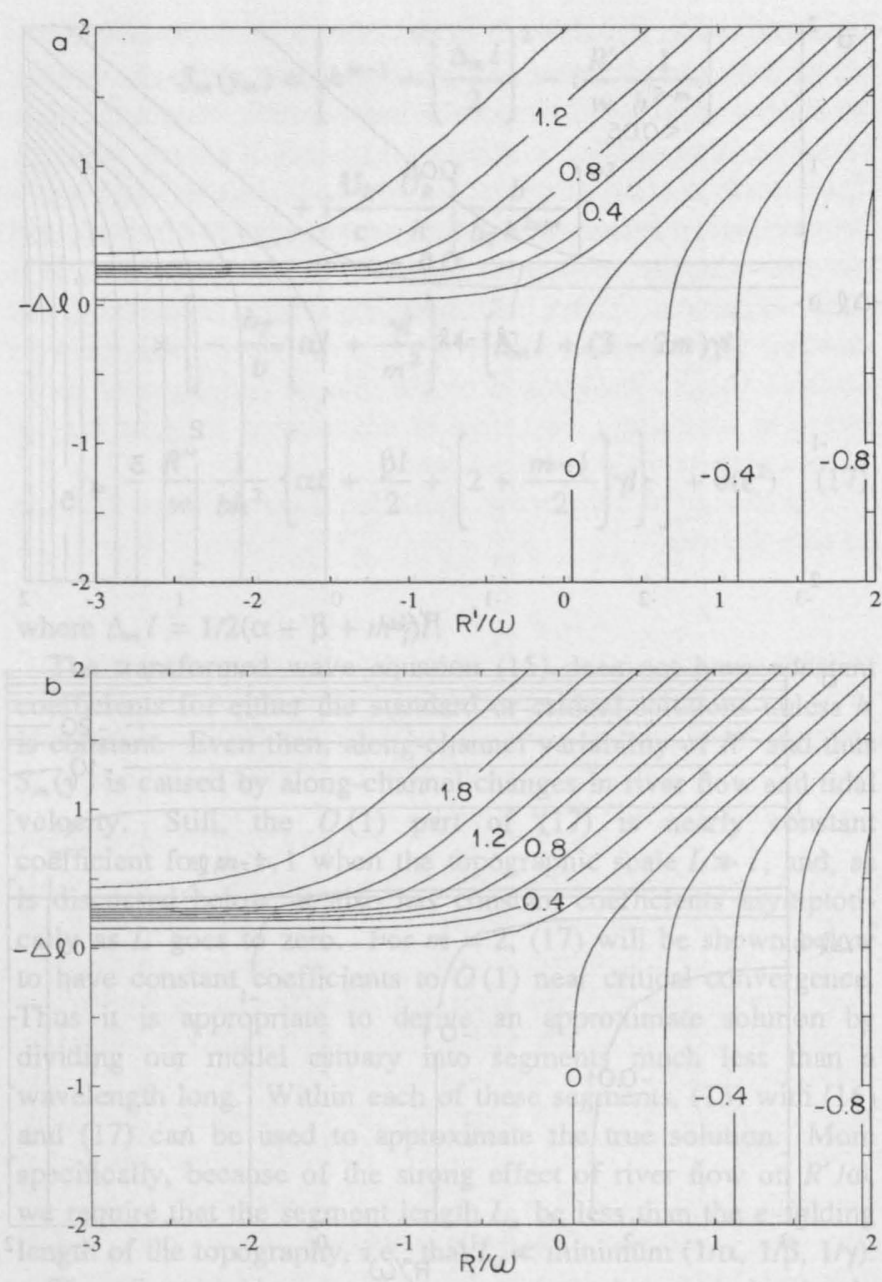

Fig. 3. Log of nondimensional wave celerity $c / c_{0}$ for convergent geometry for the $(a)$ incident and $(b)$ reflected waves as a function of the logs of $R^{\prime} / \omega$ and $-\Delta l$, with solution properties as in Figure 2. River flow and channel topography cause the reflected wave to travel faster in the strong-convergence limit. Strong friction slows incident and reflected waves equally.

sign as the effect of finite amplitude. Thus the small-amplitude inviscid wave speed $c_{0}$ is a good estimate of $c$, the actual wave speed for a tide in a channel of uniform width and depth. Increasing friction $\left(R^{\prime} / \omega \gg 1\right)$ slows the wave, but the effect is not dramatic. For weak friction the wave speed $c / c_{0}$ changes drastically at critical convergence. As $R^{\prime} / \omega$ increases, changes in $c / c_{0}$ are spread out over a larger range of $\Delta l$, a typical effect of friction. Because of the influence of $\delta l, c_{r} / c_{0}>c_{i} / c_{0}$, but this effect is subtle unless the convergence and/or river flow are strong.

The rapid wave propagation that occurs in the presence of strong convergence (or divergence) can be rationalized as follows. As $|\Delta l|$ for a section becomes very large, the cross section becomes nearly discontinuous. For a truly discontinuous change in sectional area (i.e., at the end of a channel or the intersection of a very large with a very small channel), incident and reflected waves are essentially equal, and wave propagation occurs at an apparently infinite speed; i.e., the wave phase is nearly constant along the channel leading to the junction. As is discussed below, a uniform, exponential change in cross section (even a supercritical one) does not excite a substantial reflected wave. Thus the behavior of the incident wave alone must asymptotically approach that for a standing wave at a discontinuous change of cross section. Effectively instantaneous propagation can occur only if $c / c_{0}$ becomes large.
The complex impedance function $I$ (the ratio of $\zeta / Q$ ) is determined by $q l, \delta l$ and $\Delta l$ and reveals important information about wave propagation. From (21), $I_{i}=i c^{-}$and $I_{r}=i c^{+}$at $z=0$. For weak convergence and friction, $|I|$ is essentially 1 both for the incident wave (Figure 4) and for the reflected wave as well (not shown). Not surprisingly, friction dominates $|I|$ when friction is strong, while $\Delta l$ dominates when convergence or divergence is strong. $\left|I_{i}\right|$ for the incident wave becomes very large $(Q$ small relative to $\zeta)$ as $|\Delta l|$ becomes large. In this respect also, the incident wave alone for the strong convergence/divergence case mimics a standing wave.

The phase of $I$ is the phase difference $\phi$ between $\zeta$ and $Q$ (Figure 5). Only in a deep channel (weak friction) of uniform width will $\phi$ for the incident wave approach zero, the asymptotic value for an inviscid progressive wave. Nearly inviscid incident (Figure 5 ) and reflected waves (not shown) both have $\phi \cong 0^{\circ}$ just below critical convergence and $\phi \cong 90^{\circ}$ just above, with a very abrupt change at critical convergence. Increasing friction acts to make the phase change less abrupt, as was also the case with the wave speed (Figure 3). The range of typical values of $\phi$ for major tidal species is about $10^{\circ}$ to $45^{\circ}$ for $\Delta l=0$.

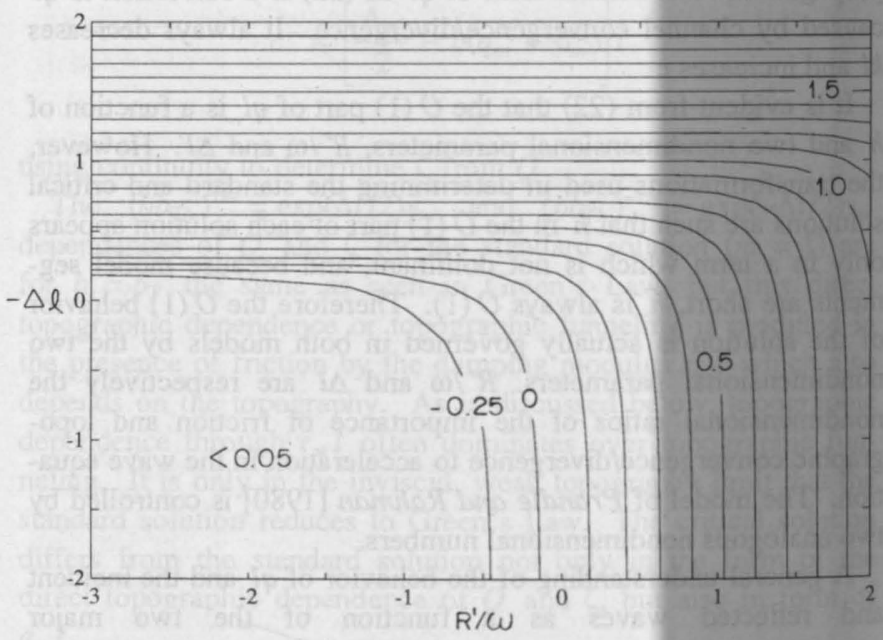

Fig. 4. Log of the impedance amplitude $|I|=|\zeta / O|$ for convergent geometry and for the incident wave as a function of the logs of $R^{\prime} / \omega$ and $-\Delta l$, with solution properties as in Figure $2 .|I|$ approaches 1 in the inviscid, weak topography limit and becomes large above critical convergence, similar to a standing wave in a channel of uniform $b$ and $h$.

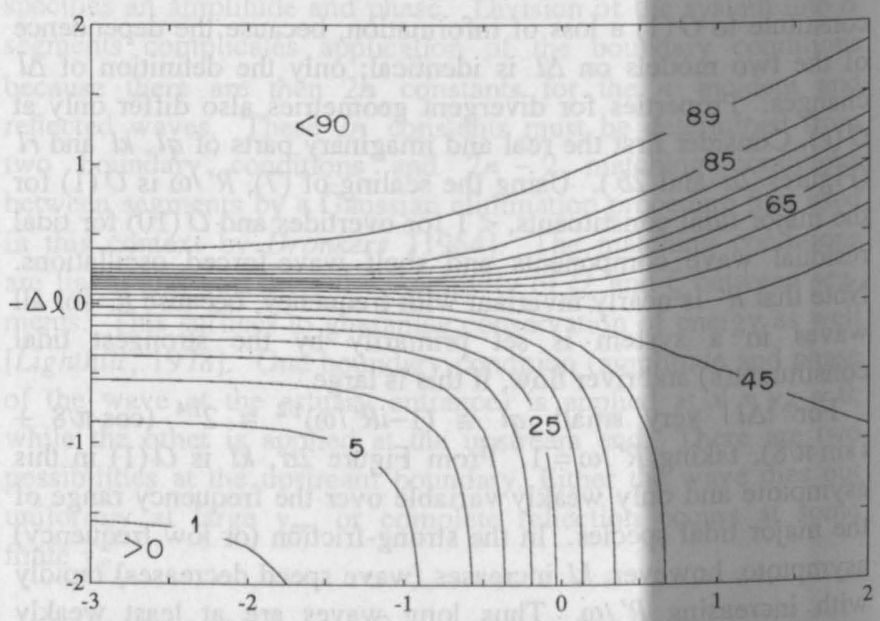

Fig. 5. Impedance phase $\phi$, the phase difference between $Q$ and $\zeta$, for convergent geometry as a function of the logs of $R^{\prime} / \omega$ and $-\Delta l$. For the incident wave, $\phi$ is $0^{\circ}$ in the inviscid, weak topography limit and $90^{\circ}$ in the strong topography limit (as in a standing wave). It is $\cong 45^{\circ}$ at critical convergence and between $10^{\circ}$ and $50^{\circ}$ for typical incident waves. 
It is in the context of the frictionally dominated, critical solution that the uniform width, strong friction model of LeBlond [1978] is relevant. LeBlond's scaling resulted in the elimination of the acceleration term in (8) and (22) as small. The result is a diffusive wave equation. More generally, any point on the $x$ axes in Figures $2 a$ and $2 b$ with $R^{\prime} / \omega>10$ fulfills the requirement of LeBlond's model that friction dominate over acceleration. From (22) and Figure $5, S$ is controlled by its imaginary part, $k l \cong-r l$, and $\phi$ is $\cong 45^{\circ}$, halfway between the values for inviscid progressive and standing waves. Friction dominates over acceleration in the vicinity of critical convergence because the effects of acceleration on $q l$ are canceled by convergence/divergence. In fact, $\phi$ in most of the lower right quadrant of Figure 5 is between $30^{\circ}$ and $50^{\circ}$. The real part of $S$ is small throughout this quadrant, because either the frequency is low, the depth is small, or the acceleration effect on the wave number is counteracted by convergence/divergence. In summary, friction commonly dominates tides in river estuaries more because convergence and acceleration cancel one another than because acceleration is inherently weak. Furthermore, waves for which acceleration effects on $S$ and $q l$ clearly cannot be ignored (e.g., high overtides) may still, through the interaction of acceleration and convergence, be frictionally dominated and mimic the behavior of low-frequency, strong-friction waves in a channel of uniform width.

The physical and mathematical bases for the sharp changes in wave properties around critical convergence can be seen from the changes in position of $S$ in the complex plane as $R^{\prime} / \omega$ and $\Delta l$ vary. Consider three cases with varying convergence rates (Figures $6 a$ to $6 c$ ). Note first that the imaginary part of $S$ is always negative, so that $k l$ and $r l$ have opposite signs. For weak, subcritical convergence with $h \cong R^{\prime} / \omega \cong 1$, the real and imaginary parts of $S$ are nearly equal (Figure $6 a$ ). This yields a $\phi$ of $\cong$ $221^{\circ}{ }^{\circ}$ with $r l$ small relative to $k l$. The critical solution is used when $R^{\prime} / w \gg 1$ and/or when acceleration and convergence/ divergence nearly cancel. In either case, $S$ is almost purely imaginary (Figure $6 b$ ). At critical convergence,

$$
\begin{gathered}
r l=-k l \quad q l=(1-i) k l \\
k l=\left[\frac{R^{\prime}}{2 \omega}\right]^{1 / 2} \\
c=c_{0}\left[\frac{2 \omega}{R^{\prime}}\right]^{1 / 2}
\end{gathered}
$$

a)

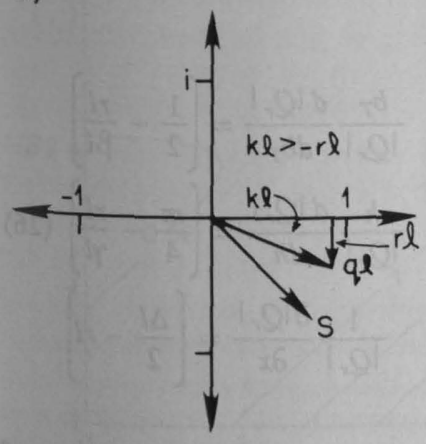

b)

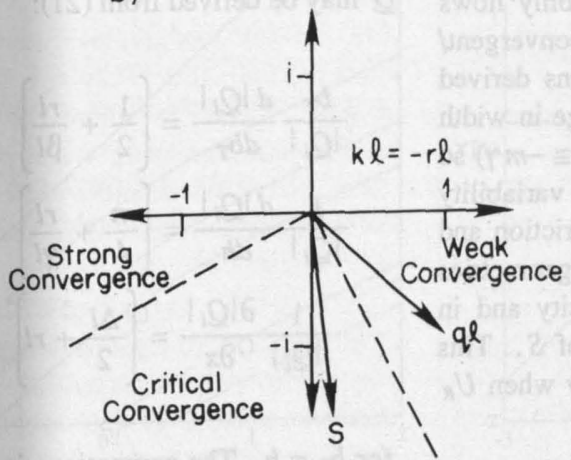

and $\phi$ is $\cong 50^{\circ}$. If friction is strong, $c<c_{0}$, but critical convergence is also possible for weak friction, in which case $c$ may be much greater than $c_{0}$. The boundaries between the standard and critical solutions used in the model are also shown in Figure $6 b$. These boundaries were chosen to be in areas where the differences between the standard and critical solutions were relatively small.

The effect of increasing $|\Delta l|$ into the supercritical convergence regime is to cause $S$ to approach the negative real axis and to increase $|S|$ (Figure $6 c$ ). This means that $q l$ is essentially imaginary, with

$$
q l \cong 0.5|\Delta l|\left[\frac{2 R^{\prime}}{\omega|\Delta l|^{2}}-i\right]
$$

Because the real part of $q l$ is small, the wave speed becomes large (Figure 3). By the same token, a real value of $\zeta$ leads to a largely imaginary value of $Q$. For $R^{\prime} / \omega=0$, the real part of $q l=i r l$, and the disturbance ceases to propagate. That is, a supercritically convergent, inviscid wave is impossible, as was noted by Lighthill [1978]. This emphasizes again the importance of friction to any realistic analysis.

\section{$O(\varepsilon)$ Properties}

The $O(\varepsilon)$ properties of $S$ stem from the convective accelerations and nonlinear, finite-amplitude friction terms. The $O(\varepsilon)$ parts of $S$ and $\delta l$ are from (15) and (17) proportional to $\mathrm{U}_{R} / c=\varepsilon \mathrm{U}_{R} / \mathrm{U}$ and are influenced by five other nondimensional parameters: $b_{T} / b, \beta l, \alpha l, \gamma l$, and $R^{\prime \prime} / \omega$. Because $\delta l$ is real, it modifies only $k l$ and, as was discussed previously, serves to slow the incident wave and accelerate the reflected wave. Because $q l$ varies as $S^{1 / 2}$, the $O(\varepsilon)$ terms in $S$ contribute to both $k l$ and $r l$.

There is one $O(\varepsilon)$ feature of the circulation that the perturbation solution presented above does not include: the increase in wave celerity associated with finite amplitude. The classical result [Lamb, 1932, article 187] for inviscid, small-amplitude waves in a uniform channel without tidal flats is that $c / c_{0} \cong 1+3 \varepsilon / 2$, or about a $15 \%$ increase in $c$ for $\varepsilon=0.1$. In the absence of overriding topographic effects, neglect of this finite-amplitude correction could lead to an error in calculating high water (even without overtide effects) of about an hour at a distance of $\lambda$ from the ocean. This would more than compensate for retardation caused by friction, assuming $R^{\prime} / \omega=1$. An approximate dispersion relation that accounts for this finite-

Fig. 6. $S l$ and $q l$ in the complex plane for $(a)$ weak convergence, $(b)$ critical convergence/strong friction, and $(c)$ strong convergence. Dotted lines in Figure $6 b$ show boundaries between normal (for weak and strong convergence) and critical solutions as employed in the model.

c)

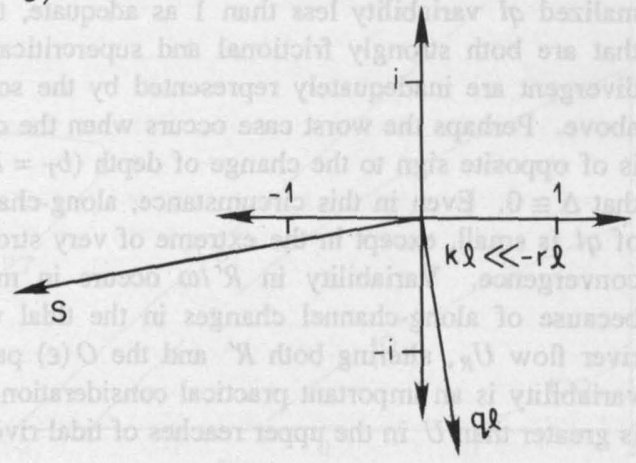


amplitude effect on $c$ can be derived as follows. The $O(\varepsilon)$ effect in question arises from nonlinearities in the convective accelerations, pressure gradient, and bed stress. Starting from a dimensional wave equation including these effects, a waveform is then assumed such that $Q \cong \exp (i(\hat{q} \hat{x}-\hat{\omega} \hat{t}))$. A quasilinearization is used to deal with the nonlinear terms. Some algebra then leads to a modified form of the $O(1)$ part of $S$ for the standard solution

$$
\begin{gathered}
S=\left[\left(1-K_{1} \varepsilon\right)-\left[\frac{\Delta l}{2}\right]^{2}-\frac{i R^{\prime}}{\omega} \frac{(1-\varepsilon)}{h}\right] \\
K_{1}=\frac{b_{T}}{b}\left[3-2 i \frac{\gamma+\alpha}{q_{0}}\right]
\end{gathered}
$$

where $q_{0}$ is $q$ without finite amplitude correction as calculated from (22). Finite amplitude thus decreases slightly the contribution of friction and acceleration to $S$. It is usually reasonable to approximate $K_{1} \cong 3 b_{T} / b$, because the $(\gamma+\alpha) / q_{0}$ part of the correction to the acceleration term is only important relative to $3 b_{T} / b$ when acceleration is itself much smaller than the convergence/divergence term. For a channel of uniform width and depth, the inviscid wave speed is $c / c_{0} \cong 1+(3 \varepsilon / 2)\left(b_{T} / b\right)$, which reduces to Lamb's [1932] result in the absence of tidal flats. No corrections are needed to the $O(\varepsilon)$ terms of $S$ (17), because these corrections would be $O(\varepsilon)^{2}$.

\section{Along-Channel Variability of the Wave Number}

The validity of the solution presented above depends on, among other factors, the validity of the assumption that the wave number is nearly constant spatially. For weak friction, the topographic convergence rate $\Delta l$ alone determines the wave number, and it is immaterial to $O(1)$ what combination of $\alpha l, \beta l$, and $\gamma l$ makes up $\Delta l$. The presence of friction, however, destroys this symmetry between depth and width changes, because $h$ appears with different powers in the acceleration and friction terms, even after the wave equation has been transformed by a change of coordinate system. Thus several different types of geometry must be considered in evaluating the variability of the wave number. $S$ (or its square root $q l$ ) is independent of $y$ to $O(1)$ for $h$ constant, except for along-channel changes in $R^{\prime} / \omega$. Another important case occurs when $b$ and $b_{T}$ are constant and equal, so that all topographic variability is caused by changes in $h$. Figures $7 a$ and $7 b$ show the normalized along-channel variability of $q l$ for the standard and critical solutions, with the realm of use of each indicated, as in Figure $6 b$. Taking a normalized $q l$ variability less than 1 as adequate, then only flows that are both strongly frictional and supercritically convergent/ divergent are inadequately represented by the solutions derived above. Perhaps the worst case occurs when the change in width is of opposite sign to the change of depth $\left(b_{T}=b, \beta \cong-m \gamma\right)$ so that $\Delta \cong 0$. Even in this circumstance, along-channel variability of $q l$ is small, except in the extreme of very strong friction and convergence. Variability in $R^{\prime} / \omega$ occurs in most geometries because of along-channel changes in the tidal velocity and in river flow $U_{R}$, altering both $R^{\prime}$ and the $O(\varepsilon)$ parts of $S$. This variability is an important practical consideration only when $U_{R}$ is greater than $U$ in the upper reaches of tidal rivers.

\section{A GENERALIZED Form OF GREEN'S LAW}

The objective of this section is to determine for all values of $R^{\prime} / \omega$ and $\Delta l$ the dependence $Q$ and $\zeta$ on $b_{T}, h$, and $x$ for both
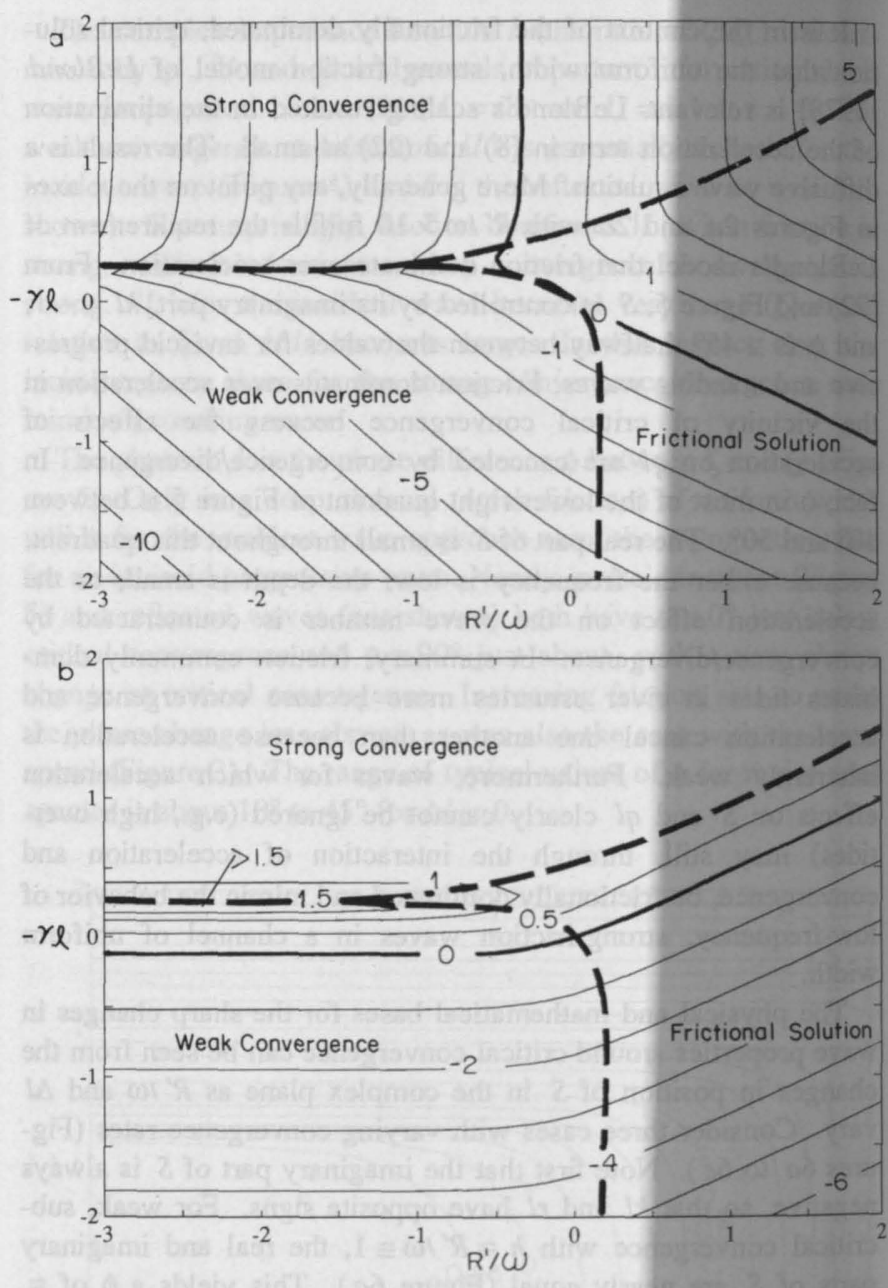

Fig. 7. Log of the normalized wave number variation $1 /|q l| \partial|q l| / \partial x$ as a function of the logs of $R^{\prime} / \omega$ and $-\gamma l$ for $(a)$ normal and $(b)$ critical solutions in a constant-width, convergent-depth channel. The solid heavy line indicates a variation of 1 , and the dotted lines delineate boundaries between normal and critical solutions. Normalized variation is $<1$ for one or the other solution, except in the strong-friction, strong-topography limit.

frictional and standard solutions, that is, to generalize Green's Law. In this regard, we are interested only in the amplitudes $|Q|$ and $|\zeta|$ and not phase information contained in the full forms of $Q$ and $\zeta$. Determination of the full topographic dependence of $|Q|$ and $|\zeta|$ requires inclusion of the topographic dependence of $r l$ as well as the effects of topographic funneling, because friction causes $|Q|$ and $|\zeta|$ to depend on the damping modulus $r l$. Algebraic expressions for the normalized topographic dependence of $Q$ may be derived from (21):

$$
\begin{array}{ll}
\frac{b_{T}}{\left|Q_{i}\right|} \frac{d\left|Q_{i}\right|}{d b_{T}}=\left[\frac{1}{2}+\frac{r l}{\beta l}\right) & \frac{b_{T}}{\left|Q_{r}\right|} \frac{d\left|Q_{r}\right|}{d b_{T}}=\left[\frac{1}{2}-\frac{r l}{\beta l}\right) \\
\frac{h}{\left|Q_{i}\right|} \frac{d\left|Q_{i}\right|}{d h}=\left(\frac{m}{4}+\frac{r l}{\gamma l}\right] & \frac{h}{\left|Q_{r}\right|} \frac{d\left|Q_{r}\right|}{d h}=\left[\frac{m}{4}-\frac{r l}{\gamma l}\right) \\
\frac{1}{\left|Q_{i}\right|} \frac{\partial\left|Q_{i}\right|}{\partial x}=\left[\frac{\Delta l}{2}+r l\right] & \frac{1}{\left|Q_{r}\right|} \frac{\partial\left|Q_{r}\right|}{\partial x}=\left[\frac{\Delta l}{2}-r l\right]
\end{array}
$$

for $b_{T}=b$. The expressions in (26) for $b_{T}$ and $h$ dependences of $Q$ give respectively $1 / 4$ and $1 / 2$ for the standard solution $(m=1)$ and $r l=0$. Deviations from these values indicate deviations from Green's Law. 
Properties for $\zeta$ differ from those for $Q$ in (26) in that minus signs are found in front of the first of the two terms in every case. Thus for example, the normalized $b_{T}$ dependence of $\zeta_{i}$ is $(-1 / 2+r l / \beta l)$. Figures 8 to 13 show normalized dependences of $Q_{i}$ and $\zeta_{i}$ for the incident wave in the standard solution $(m=1)$ as a function of $R^{\prime} / \omega$ and $\Delta l$ for convergent geometry. These plots and (26) may be viewed as a generalization of Green's Law. The plots were constructed with $b_{T}=b$ and $U_{R}=0$. The $b_{T}, h$, and $x$ dependences of the critical solution are identical to those shown in Figures 8 to 13 except as per (26) for the weak friction and convergence dependence on $h$, where a factor of $1 / 2$ appears instead of $1 / 4$. Properties of the reflected wave are identical to those for the incident wave in the weak-friction, weak-topography asymptote and may be understood from (26) and symmetry considerations discussed below in the strong-topography and strongfriction limits. Divergent geometry changes the signs of $\beta, \gamma$ and $\Delta$ in (26). Thus for example, the $b_{T}$ dependence of $Q_{i}$ for convergent geometry is the same as that of $Q_{r}$ for divergent geometry. Plots for the critical solution, divergent geometry, and reflected wave are therefore unnecessary.

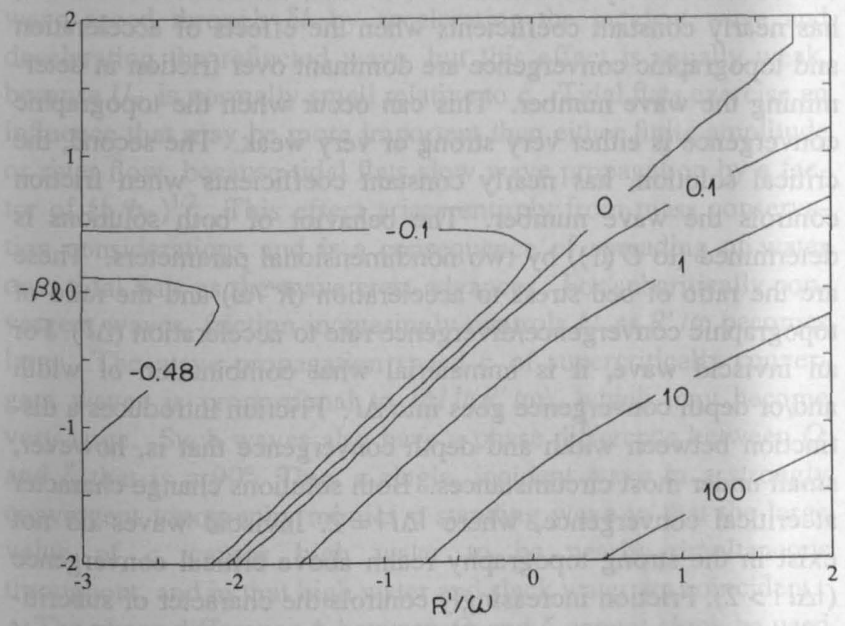

Fig. 8. Normalized $b_{T}$ variation for convergent geometry of $\zeta_{i}$ from (26) as a function of the logs of $R^{\prime} / \omega$ and $\Delta l=\beta l$ for a constant depth channel. Green's Law is the inviscid, weak-topography limit here and in Figures 9 to 11 . Symmetry considerations described in the test apply to the strongfriction and strong-topography asymptotes.

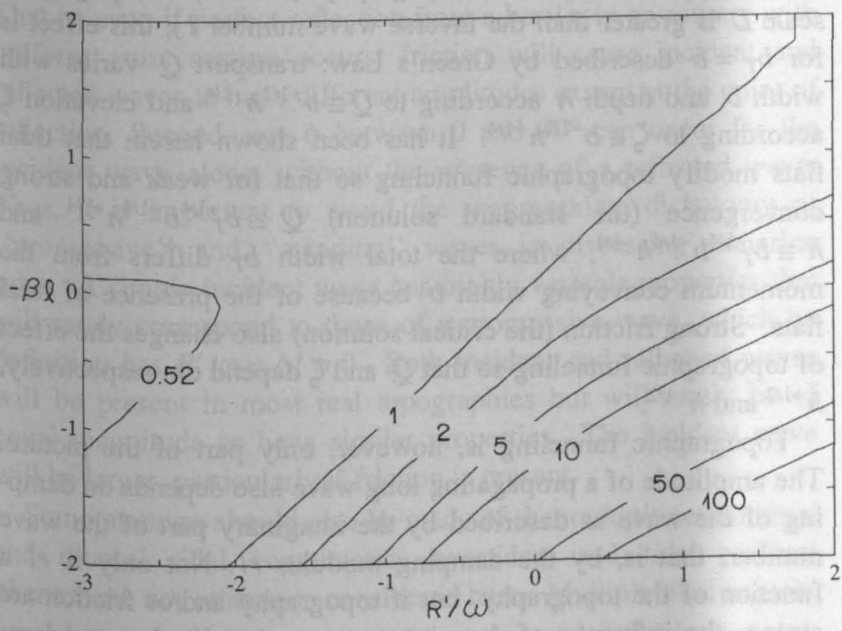

Fig. 9. Normalized $b_{T}$ variation for convergent geometry of $Q_{i}$ from (26) as a function of the logs of $R^{\prime} / \omega$ and $-\Delta l=-\beta l$ for a constant-depth channel.

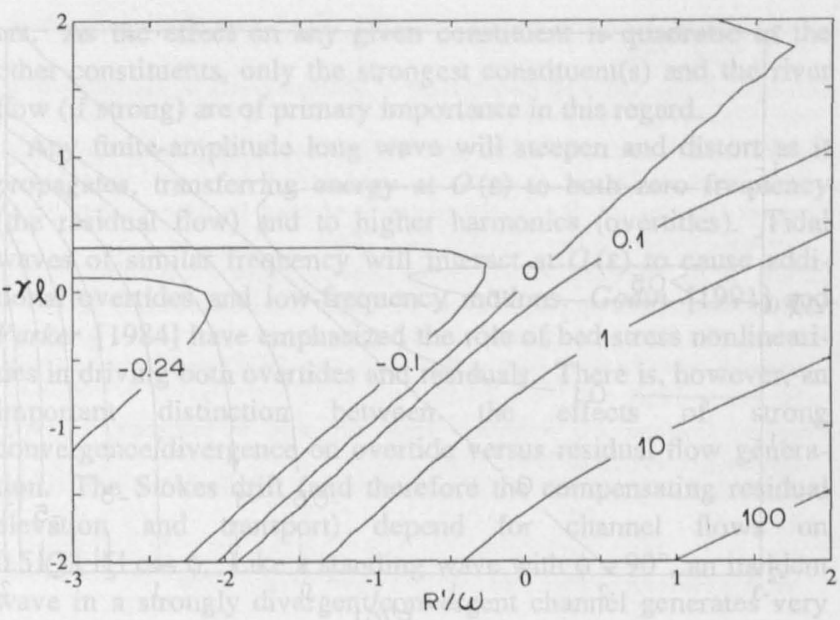

Fig. 10. Normalized $h$ variation for convergent geometry of $\zeta_{i}$ from (26) as a function of the logs of $R^{\prime} / \omega$ and $-\Delta l=-\gamma /$ for a constant-width channel.

It is useful to begin interpretation of Figures 8 to 13 by noting two asymptotic forms of $r l$. Consider first the case of weak convergence. As defined in Figure $6 a$, weak convergence implies that both convergence and friction are small relative to acceleration, and the standard solution is used in the model. From (17), $r l$ is $\cong-0.5 R^{\prime} / \omega\left(1-0.25 \Delta l^{2}\right)$ or $r l \cong-0.5 R^{\prime} / \omega$ for very weak convergence. Topographic funneling dominates the topographic response, and only a slight modification to Green's Law is seen, as in the lower left comers of Figures 8 to 11 where the $b_{T}$ variation of $Q_{i}$ and $\zeta_{i}$ approaches $\pm 1 / 2$ and the $h$ variation approaches $\pm 1 / 4$; this is also the case for reflected waves.

The strong-topography asymtotic form of $r l$ also causes the normalized topographic variations of $Q_{i}$ and $\zeta_{i}$ to have a simple form, because from (24) $r l \cong-|\Delta l| / 2$. Topographic funneling and damping are of the same magnitude. They may therefore either cancel or reinforce one another, and the normalized variation of $|Q|$ and $|\zeta|$ for $b_{T}=b$ and $U_{R}=0$ accordingly approaches 0 or \pm 1 (top left corner of Figures 8 to 11). There is also, in the absence of river flow, a symmetry between incident/reflected waves in strongly divergent/convergent channels for $Q$ and $\zeta$. For example, $\zeta_{i}$ in a convergent geometry and $\zeta_{r}$ in a divergent geometry with the same wave amplitude and value of $\Delta l$ will

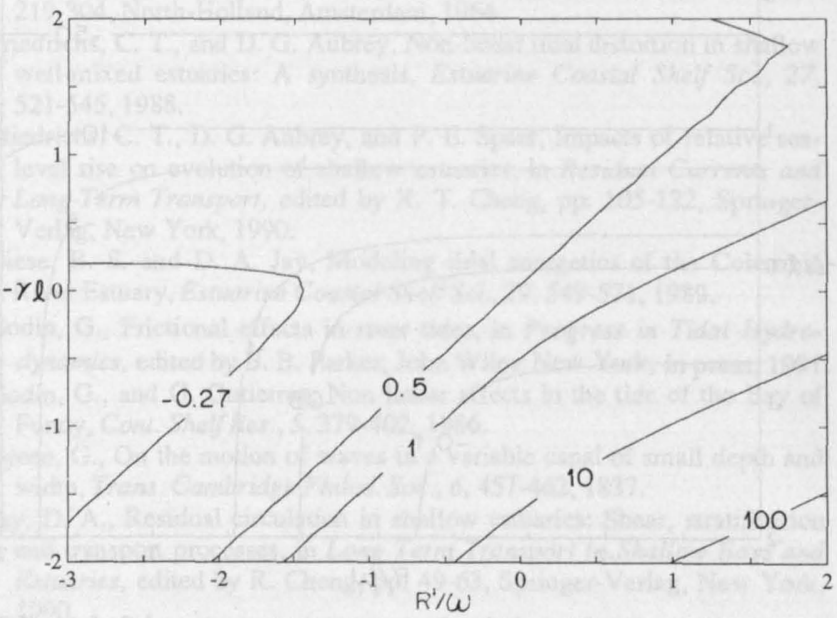

Fig. 11. Normalized $h$ variation for convergent geometry of $Q_{i}$ from (26) as a function of the logs of $R^{\prime} / \omega$ and $-\Delta l=-\gamma l$ for a constant-width channel. 


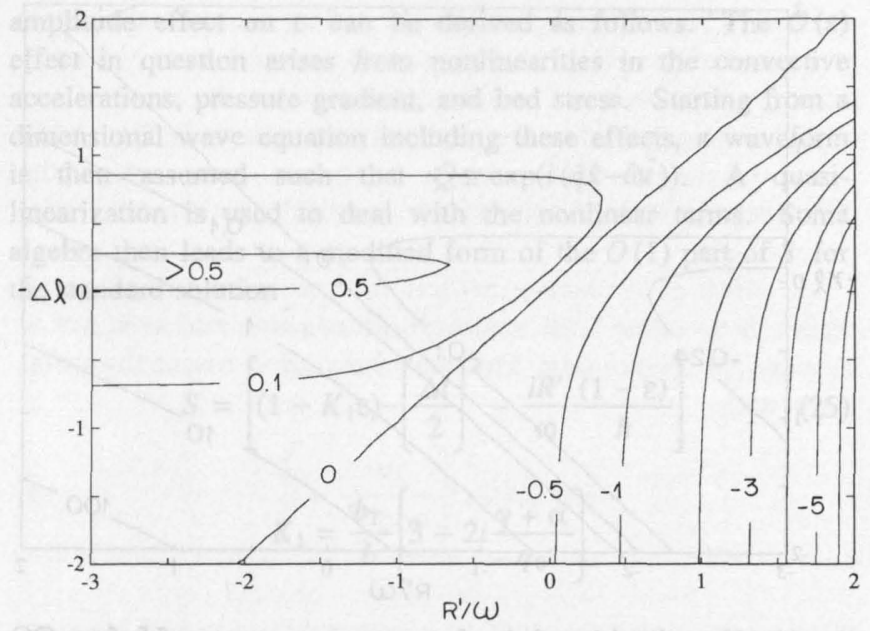

Fig. 12. Normalized $x$ variation for convergent geometry of $\zeta_{\text {, from }}(26)$ as a function of the logs of $R^{\prime} / \omega$ and $-\Delta l$. As suggested by (26) and Table (1), this plot may alternatively be viewed as representing the $x$ variation of $Q_{r}$.

exhibit the same behavior, because both waves encounter the same balance of topography and friction (Figures 12 and 13). The same is true of $Q_{i}$ in divergent geometry in relation to $Q_{r}$ in an equivalent convergent geometry, as summarized in Table 1 . This symmetry is, of course, altered by river flow.

The strong friction $b_{T}$ and $h$ variations of $|Q|$ and $|\zeta|$ are from (26) dominated by damping rather than topographic funneling for both incident and reflected waves, causing large deviations from Green's Law (lower right-hand comer of Figures 8 to 11). This is true below critical convergence even for $R^{\prime} / \omega$ of $O\left(10^{-1}\right.$ to 1$)$, which corresponds to the behavior of tide and overtide waves in typical, weakly convergent channels. Because $r l$ enters incident and reflected waves with opposite sign (equation (26)), the sign of the topographic variations in reflected wave plots is opposite to that in Figures 8 to 13 .

The above considerations concerning the behavior of $\zeta$ and $Q$ with respect to $b, h$ and $x$ apply only to individual incident or reflected waves, not to combinations thereof. Nonetheless, there are numerous circumstances where reflected waves are small and the total wave behavior is determined essentially by the incident

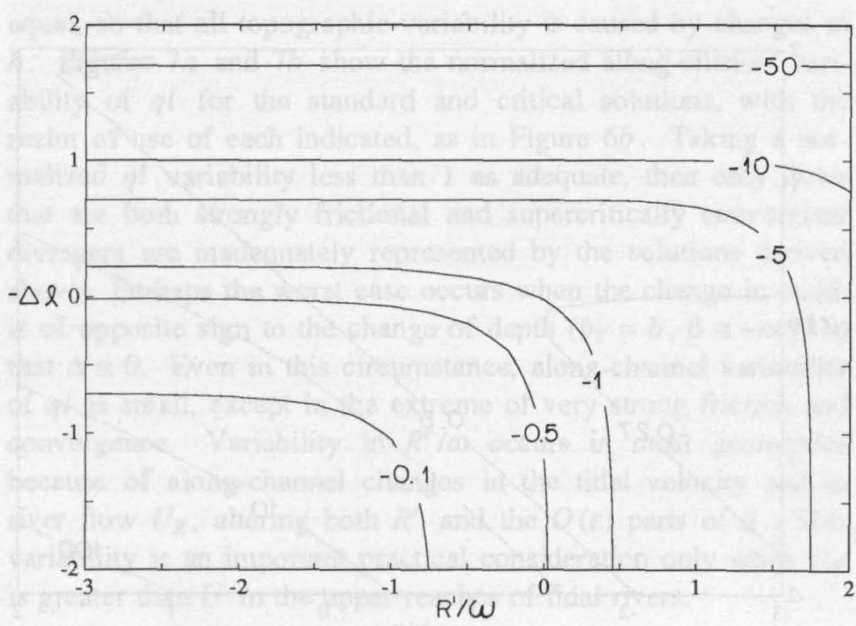

Fig. 13. Normalized $x$ variation for convergent geometry of $Q_{i}$ from (26) as a function of the logs of $R^{\prime} / \omega$ and $-\Delta l$. As suggested by (26) and Table (1), this plot may altematively be viewed as representing the $x$ variation of $\zeta_{i}$ for strong divergence.
TABLE 1. Strong-Topography Asymptotic Wave Behavior

\begin{tabular}{ccc}
\hline Wave Property & Strong Convergence & Strong Divergence \\
\hline$\zeta_{i}$ & $\left(b_{T} b h\right)^{0}$ & $\left(b_{T} b h\right)^{-1 / 2}=e^{-\mid \Delta l y}$ \\
$\zeta_{r}$ & $\left(b_{T} b h\right)^{-1 / 2}=e^{\mid \Delta l y}$ & $\left(b_{T} b h\right)^{0}$ \\
$Q_{i}$ & $\left(b_{T} b h\right)^{1 / 2}=e^{-\mid \Delta l y}$ & $\left(b_{T} b h\right)^{0}$ \\
$Q_{r}$ & $\left(b_{T} b h\right)^{0}$ & $\left(b_{T} b h\right)^{1 / 2}=e^{\mid \Delta l y}$ \\
\hline
\end{tabular}

wave. These include tides in many rivers, where friction is dominant, and very sharply convergent (in the landward direction) gulfs and bays, where as per (26) friction and topography acting together will sharply damp the reflected wave. Note also in this regard that an incident wave may have any phase difference between $Q$ and $\zeta$, even $90^{\circ}$ (Figure 5).

\section{SUMMARY AND CONCLUSIONS}

Two new solutions to the one-dimensional, tidal long-wave equation for channel flows have been derived that include the effects of friction, smooth topography of arbitrary along-channel scale, finite amplitude, river flow and tidal flats that store water but do not convey momentum. The first, the standard solution, has nearly constant coefficients when the effects of acceleration and topographic convergence are dominant over friction in determining the wave number. This can occur when the topographic convergence is either very strong or very weak. The second, the critical solution, has nearly constant coefficients when friction controls the wave number. The behavior of both solutions is determined (to $O(1)$ ) by two nondimensional parameters. These are the ratio of bed stress to acceleration $\left(R^{\prime} / \omega\right)$ and the ratio of topographic convergence/divergence rate to acceleration $(\Delta l)$. For an inviscid wave, it is immaterial what combination of width and/or depth convergence goes into $\Delta l$. Friction introduces a distinction between width and depth convergence that is, however, small under most circumstances. Both solutions change character at critical convergence, where $|\Delta l|=2$. Inviscid waves do not exist in the strong topography realm above critical convergence $(|\Delta l|>2)$. Friction increasingly controls the character of subcritically convergent waves as $R^{\prime} / \omega$ becomes large. River flow, if present, greatly increases frictional damping.

Topography influences tidal long wave propagation in channels in several ways. First, there is the direct influence of topography on the amplitude of the wave, that is, topographic funneling. For inviscid waves over weak topography (where the topographic scale $L$ is greater than the inverse wave number $l$ ), this effect is for $b_{T}=b$ described by Green's Law: transport $Q$ varies with width $b$ and depth $h$ according to $Q \cong b^{+1 / 2} h^{+1 / 4}$ and elevation $\zeta$ according to $\zeta \cong b^{-1 / 2} h^{-1 / 4}$. It has been shown herein that tidal flats modify topographic funneling so that for weak and strong convergence (the standard solution) $Q \cong b_{T}^{+1 / 4} b^{+1 / 4} h^{+1 / 4}$ and $h \cong b_{T}^{-1 / 4} b^{-1 / 4} h^{-1 / 4}$, where the total width $b_{T}$ differs from the momentum conveying width $b$ because of the presence of tidal flats. Strong friction (the critical solution) also changes the effect of topographic funneling so that $Q$ and $\zeta$ depend on, respectively, $h^{+1 / 2}$ and $h^{-1 / 2}$.

Topographic funneling is, however, only part of the picture. The amplitude of a propagating long wave also depends on damping of the wave as described by the imaginary part of the wave number, that is, by the damping modulus $r l$. Not only is $r l$ a function of the topography, but if topography and/or friction are strong, the influence of damping on wave amplitude may dominate over or balance that of topographic funneling. In this regard, three regimes may be distinguished. If friction and topog- 
raphy are both weak, then the influence of damping is small, the standard solution is appropriate, and Green's Law is asymptotically correct as friction goes to zero. If friction effects are strong (the critical solution), then the real and imaginary parts of the wave number are approximately equal, both are proportional to $R^{\prime} / \omega$, and damping dominates over topographic funneling in determining the along-channel variation in wave amplitude. Very large deviations from Green's Law occur. Finally, if topographic convergence is the dominant factor determining the wave number, $r l$ is effectively a function of $\Delta l$ only. In this case, the damping effect on wave amplitude is of the same magnitude as the direct topographic influence, which it may either reinforce or cancel, depending on the sign of $\Delta l$. Again, the result differs greatly from that of Green.

Friction, topography, the presence of tidal flats, finite amplitude (as measured by the perturbation parameter $\varepsilon$ ), and river flow all influence the real part of the wave number $k l \pm \delta l$ and the wave propagation speed $c$. For weak friction and convergence, the effect of friction in slowing the wave is often nearly balanced by finite-amplitude acceleration thereof, but the effects of finite amplitude, which scale as $O(\varepsilon)$, are otherwise smaller than the $O(1)$ effects of friction and topography. River flow modifies wave speed through $\delta l$ by accelerating the incident wave and decelerating the reflected wave, but this effect is usually weak, because $U_{R}$ is normally small relative to $c$. Tidal flats exercise an influence that may be more important than either finite amplitude or river flow, because tidal flats slow wave propagation by a factor of $\left(b / b_{T}\right)^{1 / 2}$. This effect arises entirely from mass conservation considerations and is a consequence of spreading of water onto tidal flats as the wave crest advances. For subcritically convergent waves, friction increasingly controls $k l$ as $R^{\prime} / \omega$ becomes large. The wave propagation speed $c$ of supercritically convergent waves is proportional to $|\Delta l| /\left(R^{\prime} / \omega\right)$, which may become very large. Such waves also have a phase difference between $Q$ and $\zeta$ that is $\cong 90^{\circ}$. Thus a single, incident wave in a strongly convergent topography mimics a standing wave in that the large value of $c$ causes high water to be nearly simultaneous throughout, and in that high water and slack water are coincident.

The phase difference $\phi$ between $Q$ and $\zeta$ cannot alone be used to infer anything with regard to the presence or absence of a reflected wave. For example, the argument that $\phi \cong 90^{\circ}$ implies the existence of a standing wave with equal incident and reflected waves is fallacious for two reasons. First, friction is important in all estuarine systems and dominant in most shallow estuaries. That is, even if perfect reflection from a barrier in an estuary with uniform cross section occurs, friction will cause incident and reflected waves to have different amplitudes except at the point of reflection. Second, any $\phi$ between 0 and $90^{\circ}$ can occur for the incident wave alone, without the presence of a reflected wave. Thus, it is important to avoid the inappropriate dichotomy of "progressive" and "standing" waves in discussing estuarine tides. A simple incident wave has highly variable properties that will rarely correspond to those of a progressive wave, which by definition has $R^{\prime} / \omega=\Delta l=0$. Both incident and reflected waves will be present in most real topographies but will rarely be of equal magnitude or have similar properties. The incident wave will be larger, particularly if friction is present.

Some mention should also be made of the multiple semidiurnal and diumal tidal constituents, overtides, and tidal residual phenomena not otherwise considered here. Multiple tidal constituents have not been incorporated into the model at this time, but their inclusion is straightforward, as they interact at $O(1)$ only through the $P_{3}$ term in (9), so that each constituent damps all oth- ers. As the effect on any given constituent is quadratic in the other constituents, only the strongest constituent(s) and the river flow (if strong) are of primary importance in this regard.

Any finite-amplitude long wave will steepen and distort as it propagates, transferring energy at $O(\varepsilon)$ to both zero frequency (the residual flow) and to higher harmonics (overtides). Tidal waves of similar frequency will interact at $O(\varepsilon)$ to cause additional overtides and low-frequency motions. Godin [1991] and Parker [1984] have emphasized the role of bed stress nonlinearities in driving both overtides and residuals. There is, however, an important distinction between the effects of strong convergence/divergence on overtide versus residual flow generation. The Stokes drift (and therefore the compensating residual elevation and transport) depend for channel flows on $0.5|Q||\zeta| \cos \phi$. Like a standing wave with $\phi \cong 90^{\circ}$, an incident wave in a strongly divergent/convergent channel generates very little Stokes drift or residual. This is by no means the case with overtides in the same geometry, as these are quadratic in $Q$ or $\zeta$, but do not depend on $\phi$. Strong convergence/divergence may be expected to have an important effect on the phase of the overtide relative to the basic tidal wave. In this regard, topography may either augment or compete with the effects of tide flats. Speer and Aubrey [1985] have demonstrated that the presence of broad tidal flats changes the phase relationship between the basic tidal wave and its first overtide, such that broad, shallow systems are ebb dominant (overtide reinforces the ebb current) rather than flood dominant, as is typically the case for narrow estuaries. C. Friedrichs and O. Madsen (manuscript in preparation, 1991) have interpreted long wave behavior in channels with tidal flats in terms of a modified form of $\varepsilon$ (which becomes negative in the presence of broad tidal flats) and a frictional length scale. This behavior may also be understood by considering the additional inhomogeneous forcing terms introduced into the overtide wave equation by the presence of tidal flats. An extension of the present model will consider this problem.

Acknowledgments. This work was supported by NSF grant OCE8711790. G. Godin reviewed the manuscript and offered numerous interesting suggestions. Lyn Sylwester assisted in preparation of the figures.

\section{REFERENCES}

Dorrestein, R, Amplification of long waves in bays, Eng. Prog. Univ. of Fla., 15, 1-21, 1961.

Dronkers, J. J., Tidal Computations in Rivers and Coastal Waters, pp. 219-304, North-Holland, Amsterdam, 1964.

Friedrichs, C. T., and D. G. Aubrey, Non-linear tidal distortion in shallow well-mixed estuaries: A synthesis, Estuarine Coastal Shelf Sci., 27, 521-545, 1988

Friedrichs, C. T., D. G. Aubrey, and P. E. Speer, Impacts of relative sealevel rise on evolution of shallow estuaries, in Residual Currents and Long-Term Transport, edited by R. T. Cheng, pp. 105-122, SpringerVerlag, New York, 1990.

Giese, B. S. and D. A. Jay, Modeling tidal energetics of the Columbia River Estuary, Estuarine Coastal Shelf Sci., 29, 549-571, 1989.

Godin, G., Frictional effects in river tides, in Progress in Tidal Hydrodynamics, edited by B. B. Parker, John Wiley New York, in press, 1991. Godin, G., and G. Gutierrez, Non-linear effects in the tide of the Bay of Fundy, Cont. Shelf Res., 5, 379-402, 1986.

Green, G., On the motion of waves in a variable canal of small depth and width, Trans. Cambridge Philos. Soc., 6, 457-462, 1837.

Jay, D. A., Residual circulation in shallow estuaries: Shear, stratification and transport processes, in Long Term Transport in Shallow Bays and Estuaries, edited by R. Cheng, pp. 49-63, Springer-Verlag, New York, 1990.

Jay, D. A., Intemal asymmetry and anharmonicity in estuarine flows, in Progress in Tidal Hydrodynamics, edited by B. B. Parker, pp. 521-543, John Wiley, New York, 1991. 
Lamb, H., Hydrodynamics, pp. 273-282, Cambridge University Press, New York, 1932.

LeBlond, P. H., On tidal propagation in shallow rivers, J. Geophys. Res., $83,4717-4721,1978$.

Lighthill, J., Waves in Fluids, pp. 89-136, Cambridge University Press, New York, 1978.

Longuet-Higgins, M. S., On the transport of mass by time-varying ocean currents, Deep Sea Res., 16, 431-447, 1969.

Parker, B. B., Frictional effects on the tidal dynamics of a shallow estuary, Ph.D. thesis, 292 pp., Johns Hopkins Univ., Baltimore, Md., 1984.

Perroud, P., The propagation of tidal waves into channels of gradually varying cross-section, Inst. of Eng. Res., Ser. 89, 27 pp., Univ. of Calif., Berkeley, 1958.

Prandle, D., and M. Rahman, Tidal response in estuaries, J. Phys. Oceanogr., 10, 1552-1573, 1980.

Proudman, J., Dynamical Oceanography, 409 pp., John Wiley, New York, 1953.

Speer, P. E., Tidal distortion in shallow estuaries, Ph.D. thesis, 210 pp., Woods Hole Oceanogr. Inst., Woods Hole, Mass., 1984.
Speer, P. E., and D. Aubrey, A study of non-linear tidal propagation in shallow inlet/estuarine systems, II, Theory, Estuarine Coastal Shelf Sci., 21, 206-240, 1985.

Stokes, G. G., On the theory of oscillatory waves, Trans. Cambridge Philos. Soc., 8, 441-455, 1847.

Whitham, G. B., Linear and Non-Linear Waves, pp. 2-13, WileyInterscience, New York, 1974.

Zimmerman, J. F. T., On the Euler-Lagrange transformation and the Stokes' drift in the presence of oscillatory and residual currents, Deep Sea Res., Part A, 26, 505-520, 1978.

Zimmerman, J. F. T., The tidal whirlpool: A review of horizontal dispersion by tidal and residual currents, Neth. J. Sea Res., 20, 133-154, 1986.

D. A. Jay, Geophysics Program, AK-50, University of Washington, Seattle, WA 98195.

(Received August 13, 1990; revised March 11, 1991; accepted April 23, 1991) 